\title{
Two-Dimensional Non-Layered Materials: Synthesis, Properties and Applications
}

\author{
Feng Wang, Zhenxing Wang, * Tofik Ahmed Shifa, Yao Wen, Fengmei Wang, \\ Xueying Zhan, Qisheng Wang, Kai Xu, Yun Huang, Lei Yin, Chao Jiang, and Jun He*
}

Holding novel physical properties, high flexibility and strong integration ability with Si-based electronic devices, two-dimensional (2D) non-layered materials have received considerable attentions in recent years. To achieve the 2D anisotropic growth, various strategies have been developed. And a variety of applications have been demonstrated. This review provides an overview on the recent progress of this material family. The scope will cover the preparation strategies, including dry methods and wet chemical approaches, as well as the applications in catalysis, energy conversion and storage, optoelectronic devices and topological crystalline insulators. Conclusion and future perspectives are also given.

\section{Introduction}

Mophology regulation has been one of the most important issues in the field of nanomaterials. This is stimulated by the fact that the properties and the final performances of nanomaterials are strongly dependent on their specific structures. For example, the density of charge states changes from discrete levels for zero-dimensional quantum dots to a saw-like quasicontinuum in one-dimensional nanorods, and finally to step-like quasicontinuum in two-dimension nanoplates. ${ }^{[1]}$ Particularly, two dimensional (2D) structures generally refer to the plates with ultrathin thickness even down to only few-atom

Prof. Z. Wang, Prof. C. Jiang, Prof. J. He CAS Center for Excellence in Nanoscience CAS Key Laboratory of Nanosystem and Hierarchical Fabrication

National Center for Nanoscience and Technology Beijing 100190, China

E-mail:wangzx@nanoctr.cn; hej@nanoctr.cn

F. Wang, T. A. Shifa, Y. Wen, F. M. Wang, X. Zhan,

K. Xu, Y. Huang, L. Yin

CAS Center for Excellence in Nanoscience

CAS Key Laboratory of Nanosystem and Hierarchical Fabrication

National Center for Nanoscience and Technology

Beijing 100190, China

F. Wang, T. A. Shifa, Y. Wen, F. M. Wang, X. Zhan,

K. Xu, Y. Huang, L. Yin

University of Chinese Academy of Sciences

Beijing 100049, P. R. China

Dr. Q. Wang

Department of Electrical and Computer Engineering

National University of Singapore

117576, Singapore layers. Since charge carriers, heat and photon transports are strongly confined in a 2D plane, unusual physical phenomena such as 2D electron gas $(2 \mathrm{DEG})^{[2]}$ and quantum hall effects ${ }^{[3]}$ will occur. And the 2D geometry may also bring new applications like charge density wave (CDW), ${ }^{[4]}$ and valley-related spintronics. ${ }^{[5]}$ In addition, due to the dramatically ehnhanced surface states, 2D structures have shown great promising applications in sensing, catalysis, energy storage and conversion, topological crystalline insulators (TCIs), bio-imaging and magnetic recording, etc. $^{[6]}$ More importantly, unlike the other low dimensional structures, the 2D geometry show great compatibility with current thin film manufacture techniques, hence are suitable for monolithic integration with traditional electronic materials like silicon and can be placed on variable substrates, even flexiable ones, which undoubtedly promote the practical applications. These advantages mentioned above and others are the reason for the extensive research attention that $2 \mathrm{D}$ structures have received in the past twelve years.

Due to the weak van der Waals interaction between the vertically stacked layers, the flakes of layered materials with desired thickness can be easily exfoliated from their bulk crystals and exhibit novel properties, such as layer number-dependent band structures, optical properties, and valley-polarization. ${ }^{[7,8]}$ So far, a great number of layered materials have been extensively studied, including (1) Group IVA elements like Graphene, Silicene, and Gemanene, (2) Black phosphorene, (3) $h$-BN, (4) Transition metal dichalcogenides like $\mathrm{MoS}_{2}, \mathrm{MoSe}_{2}, \mathrm{MoTe}_{2}$, $\mathrm{WS}_{2}, \mathrm{WSe}_{2}$, and $\mathrm{WTe}_{2}$, (5) III-VI compounds like $\mathrm{In}_{2} \mathrm{Se}_{3}, \mathrm{GaS}$, GaSe, and GaTe, (6) V-VI compounds like $\mathrm{Bi}_{2} \mathrm{Se}_{3}$ and $\mathrm{Bi}_{2} \mathrm{Te}_{3} \cdot{ }^{[7,8]}$ Holding the specific properties of their own and more importantly benefitting from their unique 2D structures, this family of materials has been demonstrated with various promising applications in electronics, optoelectronics, catalysis, energy conversion and storage, sensing and so on.

The research on 2D layered materials has achieved a big success. However, their species and amounts are limited after all and much more functional materials with superior characteristics have crystal structures of chemical bonding in three dimensions (rather than the two dimensions of layered materials, hence we call them non-layered materials). For examples typical III-V group semiconductors have both high carrier mobility and direct band gaps. Taking the plenty advantages of $2 \mathrm{D}$ geometry into consideration, it would be

DOI: $10.1002 / \mathrm{adfm} .201603254$ 
a natural attempt to see what new insight it would bring to us when synthesizing non-layered materials into 2D structures. In addition, 2D non-layered materials with both the novel properties of their bulks and the 2D chracteristics can be necessary complement to 2D layered materials. As a consequence, the research on 2D non-layered materials has attracted growing attentions and do show some beneficial effects. For example, the surfaces of 2D non-layered materials are filled with dangling bonds, which is not the case in layered materials, enabiling their surface highly chemically active and enhancing their capability of catalysis, sensing and carriers transfer. In another instance, $2 \mathrm{D} \mathrm{Pb}_{1-\mathrm{x}} \mathrm{Sn}_{\mathrm{x}} \mathrm{Te}$ nanoplates have enabled the experimental observation of the crystalline symmetry-protected topological surface properties. ${ }^{[9]}$ However, in the realistic experiments, it is not an easy job to downsize the vertical dimension while keeping the others unchanged (or changed a little) due to the intrinsic isotropic chemically bonded nature. To realize the $2 \mathrm{D}$ anisotropic growth of non-layered materials, a number of synthesis strategies have been developed, including both wet chemistry and dry methods..$^{[7,10]}$ No matter which methods it takes, the thermodynamic equilibrium state must be broken and the controllability of kinetics must be artificially introduced to stimulate the 2D anisotropic growth. Through these strategies, on one hand, novel physical and chemical properties based on the 2D confined structures can be obtained. On the other hand, 2D structures can be facilely integrated into Si-based microelectronic devices because of the high compatibility with current microelectronic processing, which is important for the practical applications in the future.

Recently, researchers, including our group, have made a big breakthrough in the synthesis and applications of 2D nonlayered materials. Here, we aim to give an overview on the recent progress of 2D non-layered materials, including metals, metal oxides, metal chalcogenides, topological crystalline insulators, organic-inorganic hybrid perovskites, and others. We first summarize various types of 2D non-layered materials and discuss the related synthesis methods, including dry methods and wet-chemical approaches. Then we emphasize on some promising applications in catalysis, energy storage and conversion, optoelectronics, and topological crystalline insulators. Finally, conclusion and future perspectives are given.

\section{Materials Category, Synthesis Mechanism, and Methods}

\subsection{Materials Category}

Up to now, a variety of non-layered materials have been synthesized successfully into 2D structures, including metals, ${ }^{[11-16]}$ metal oxides, ${ }^{[17-21]}$ metal chalcogenides, ${ }^{[22-29]}$ topological crystalline insulators, ${ }^{[9,30-33]}$ organic-inorganic hybrid perovskites, ${ }^{[34-36]}$ and others. With this special 2D geometry, they have been studied as photodetectors, low-dissipation logic devises, catalysts for water splitting and $\mathrm{CO}$ oxidation, and electrodes for Li-ion batteries etc. Table 1 briefly summarizes the types of materials, synthesis methods, dimensions, applications and representative performances.

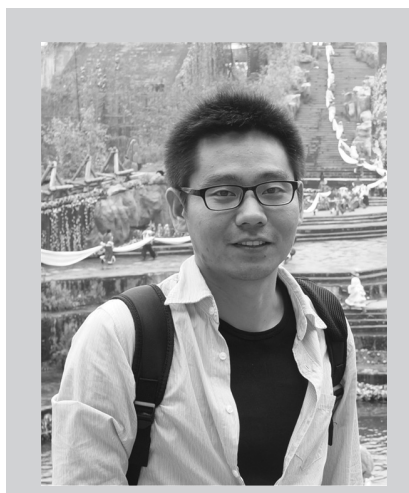

Mr. Feng Wang received his Bachelor's degree from the Department of Materials Physics, Yanshan University, in 2010. Then he obtained his Master's degree in engineering from Zhejiang University in 2013. After that, he worked as a Research Assistant in the Department of Mechanical and Automation Engineering, the Chinese University of Hong Kong. Now he is a PhD candidate under the supervision of Prof. He in the National Center for Nanoscience and Technology (NCNST). His current research is developing two-dimensional materials for electronic and optoelectronic applications.

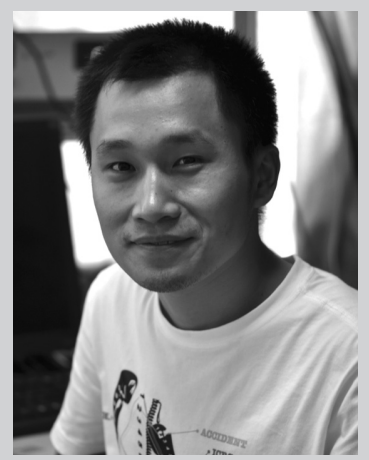

Dr. Zhenxing Wang received his BS and PhD degrees from University of Science and Technology of China (USTC) in 2002 and 2009, respectively. After a twoyear postdoctoral work at Peking University, he joined the National Center for Nanoscience and Technology (NCNST) in 2011, where he currently is an associate Professor. His current research interests focus on low dimensional materials and optoelectronic devices.

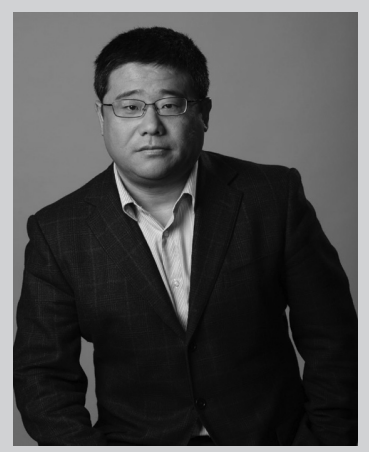

Dr. Jun He received his $\mathrm{PhD}$ in Semiconductor Physics from the Institute of Semiconductors, Chinese Academy of Sciences (CAS), in 2003. Then he worked successively at Applied Physics Department of Technische Universiteit Eindhoven, Netherlands, Material Department of University of California, Santa Babara, and California NanoSystem Insitute (CNSI), University of California, Los Angeles, USA. He joined the "100-Talents" Program of CAS in Nov. 2010 and became a Full Professor of the National Center for Nanoscience and Technology since then. His main research interest is the synthesis, characterization, and devices of low dimensional semiconductor materials.

\subsection{Synthesis Thermodynamics and Kinetics}

Non-layered materials, as the name shows, are strongly kept together by chemical bonds in all the three dimensions. According to the energy differences between their crystalline 
www.MaterialsViews.com

Table 1. Summary of 2D non-layered materials.

\begin{tabular}{|c|c|c|c|c|c|c|c|}
\hline \multirow[t]{2}{*}{ 2D nanomaterials } & & \multirow[t]{2}{*}{ Synthesis method } & \multicolumn{2}{|c|}{ Dimensions } & \multirow[t]{2}{*}{ Applications } & \multirow[t]{2}{*}{ Performances } & \multirow[t]{2}{*}{ Ref. } \\
\hline & & & Lateral dimensions & Thickness (nm) & & & \\
\hline Lead tri-halide perovskites & $\mathrm{CH}_{3} \mathrm{NH}_{3} \mathrm{Pbl}_{3}$ & $\begin{array}{c}\text { Template-directed } \\
\text { CVD }\end{array}$ & $5-30 \mu \mathrm{m}$ & $10-600$ & & & [34] \\
\hline \multirow[t]{3}{*}{$\mathrm{TCls}$} & $\mathrm{SnTe}$ & CVD & $\sim 2-20 \mu \mathrm{m}$ & $\sim 100-350$ & & & [31] \\
\hline & $\mathrm{Pb}_{1-x} \mathrm{Sn}_{x} \mathrm{Se}$ & vdWE & $20-50 \mu \mathrm{m}$ & $15-45$ & Photodetectors & $5.95 \mathrm{~A} \mathrm{~W}^{-1}(473 \mathrm{~nm})$ & [32] \\
\hline & $\mathrm{Pb}_{1-x} \mathrm{Sn}_{x} \mathrm{Te}$ & vdWE & $10-20 \mu \mathrm{m}$ & $\sim 40$ & & & [9] \\
\hline \multirow[t]{7}{*}{ Metal } & $\mathrm{Ag}$ & $\begin{array}{l}2 \mathrm{D} \text { oriented } \\
\text { attachment }\end{array}$ & $\sim 4 \mu \mathrm{m}$ & $<10$ & & & [42] \\
\hline & $\mathrm{Au}$ & TDS (GO) & $200-500 \mathrm{~nm}$ & $\sim 2.4$ & & & [12] \\
\hline & $\mathrm{Pd}$ & CSS (CO) & $20-160 \mathrm{~nm}$ & $\sim 7.8$ & Photothermal therapy & $\begin{array}{l}\text { 100\% kill (liver cancer } \\
\text { cell, } 808 \mathrm{~nm}, 5 \mathrm{~min} \text { ) }\end{array}$ & [43] \\
\hline & $\mathrm{Fe}$ & $\begin{array}{l}\text { Sealing graphene } \\
\text { holes }\end{array}$ & $\sim 2-3 \mathrm{~nm}$ & $\sim 0.26$ & & & [13] \\
\hline & $\mathrm{Rh}$ & $\begin{array}{l}\text { Solvothermal } \\
\text { method }\end{array}$ & $500-600 \mathrm{~nm}$ & $\sim 0.36$ & Catalysis & $\begin{array}{l}\quad>99.9 \% \text { coversion for } \\
\text { hydrogeneration (phenol) \& } \\
\text { hydroformylation (1-octene) }\end{array}$ & [44] \\
\hline & $\mathrm{Ru}$ & $\begin{array}{l}\text { Hydrothermal } \\
\text { method }\end{array}$ & $13-27 \mathrm{~nm}$ & $\sim 1.3-3.6$ & $\mathrm{CO}$ conversion & & [45] \\
\hline & Co & $\begin{array}{l}\text { Ligand-confined } \\
\text { growth }(48 \mathrm{~h})\end{array}$ & $\sim 400-500 \mathrm{~nm}$ & $\sim 0.84$ & $\mathrm{CO}_{2}$ reduction & $\begin{array}{l}0.24 \mathrm{~V} \text { (overpotential), } \\
10.59 \mathrm{~mA} \mathrm{~cm}^{-2}, 90.1 \% \\
\text { (selectivity) }\end{array}$ & [83] \\
\hline \multirow[t]{8}{*}{ Metal oxides } & $\mathrm{NiO}$ & TDS (Ni(OH)3) & $>1 \mu \mathrm{m}$ & $\sim 1.16$ & & & [50] \\
\hline & $\mathrm{TiO}_{2}$ & CSS \& exfoliation & $\sim 200 \mathrm{~nm}$ & $\sim 2.7-3.3$ & Photodetectors & $\mathrm{I}_{\text {on }} / \mathrm{I}_{\text {off }} \sim 2(325 \mathrm{~nm})$ & [20] \\
\hline & $\mathrm{CeO}_{2}$ & CSS & $\sim 400 \mathrm{~nm}$ & $\sim 0.6$ & CO conversion & $50 \%$ conversion at $131^{\circ} \mathrm{C}$ & [21] \\
\hline & $\mathrm{Eu}_{2} \mathrm{O}_{3}$ & $\begin{array}{l}\text { Assembly of } \\
\text { nanowires }\end{array}$ & $\mathrm{W}: 200 \mathrm{~nm}-2 \mu \mathrm{m}$ & $\sim 3.8$ & & & [18] \\
\hline & & & $\mathrm{L}: 400 \mathrm{~nm}-10 \mu \mathrm{m}$ & & & & \\
\hline & $\mathrm{SnO}_{2}$ & $\begin{array}{l}\text { Solvothermal } \\
\text { method }\end{array}$ & $>100 \mathrm{~nm}$ & $\sim 0.66$ & CO conversion & $50 \%$ conversion at $165^{\circ} \mathrm{C}$ & [63] \\
\hline & $\mathrm{Cd}_{2} \mathrm{O}_{3}$ & Colloidal synthesis & $\sim 200 \mathrm{~nm}$ & $<1$ & & & [52] \\
\hline & $\mathrm{ZnO}$ & CSS \& exfoliation & $1-10 \mu \mathrm{m}$ & $\sim 3.35$ & Photodetectors & $\mathrm{I}_{\text {on }} / \mathrm{I}_{\text {off }} \sim 2(325 \mathrm{~nm})$ & [20] \\
\hline \multirow[t]{9}{*}{ Metal chalcogenides } & $\mathrm{PbS}$ & $\begin{array}{l}2 \mathrm{D} \text { oriented } \\
\text { attachment }\end{array}$ & $>1 \mu \mathrm{m}$ & $\sim 2.2$ & Photodetectors & $0.472 \mathrm{~A} \mathrm{~W}(532 \mathrm{~nm})$ & [22] \\
\hline & $\mathrm{PbS}$ & vdWE & $10-20 \mu \mathrm{m}$ & $\sim 20$ & Phototransistor & $1621 \mathrm{~A} \mathrm{~W}^{-1}(800 \mathrm{~nm})$ & [40] \\
\hline & $\mathrm{PbSe}$ & Colloidal synthesis & $\sim 200 \mathrm{~nm}$ & $\sim 4.3 \pm 0.5$ & & & [54] \\
\hline & $\mathrm{CdS}$ & CSS \& exfoliation & $100-300 \mathrm{~nm}$ & $\sim 4$ & $\mathrm{H}_{2}$ evolution reaction & $1.38 \%$ (quantum efficiency) & [61] \\
\hline & CuS & $\begin{array}{l}\text { Soft colloidal } \\
\text { templating }\end{array}$ & $453 \pm 6 \mathrm{~nm}$ & $\sim 3.2$ & Li-ion battery & $642 \mathrm{~mA} \mathrm{~g}^{-1}\left(0.2 \mathrm{~A} \mathrm{~g}^{-1}\right)$ & [23] \\
\hline & CuSe & $\begin{array}{l}\text { Hot-injection } \\
\text { method }\end{array}$ & $\sim 0.6-1.6 \mu \mathrm{m}$ & $\sim 5.2$ & & & [29] \\
\hline & $\mathrm{ZnSe}$ & $\begin{array}{l}\text { Liquid phase } \\
\text { exfoliation }\end{array}$ & $\sim 500 \mathrm{~nm}$ & $\sim 0.91$ & Photocatalysis & $42.5 \%$ (IPCE) & [26] \\
\hline & $\mathrm{CdTe}$ & Colloidal synthesis & $20-100 \mathrm{~nm}$ & $1.62-3.56$ & & & [1] \\
\hline & $\mathrm{CoSe}_{2}$ & $\begin{array}{l}\text { Liquid phase } \\
\text { exfoliation }\end{array}$ & $>100 \mathrm{~nm}$ & $\sim 1.4$ & & & [84] \\
\hline \multirow[t]{2}{*}{ Others } & $\delta$-FeOOH & $\begin{array}{l}\text { Topochemical } \\
\text { transformation }\end{array}$ & $>200 \mathrm{~nm}$ & $\sim 1.2$ & ferromagnetics & $7.5 \mathrm{emu} \mathrm{g}^{-1}$ & [85] \\
\hline & $\mathrm{Si}$ & TDS & $\sim 400 \mathrm{~nm}$ & $\sim 3.5$ & Li-ion battery & $\sim 600 \mathrm{~mA} \mathrm{~h} \mathrm{~g}^{-1}$ & [86] \\
\hline
\end{tabular}

TCIS: Topological crystalline insulators; CVD: chemical vapor deposition; vdWE: van der Waals Epitaxy; TDS: template-directed synthesis; CSS: confined space synthesis 

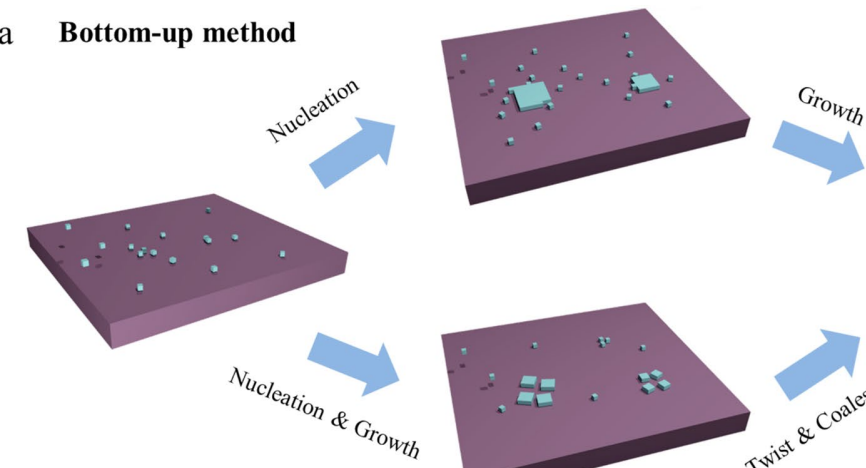

www.MaterialsViews.com

b Combined strategy

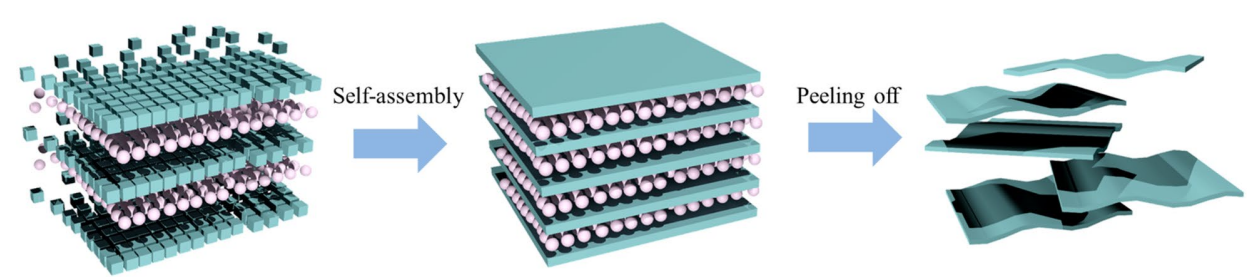

Figure 1. a) Schematic diagram of the bottom-up synthesis method using van der Waals epitaxy (vdWE) as an example. The red sheets, green dots and green plates represent the substrates, adatoms, and 2D non-layered nanomaterials, respectively. b) Schematic diagram of the 'combined strategy' with the green cubes, red balls, and green planes representing the precursors of the non-layered material, the organic spacers, and the obtained $2 \mathrm{D}$ non-layered materials, respectively.

faces, the crystals are usually found with particular morphologies. For the same reason, they thermodynamically tend to form structures that are capable of showing their symmetries (which actually corresponds to the lowest total energy of the system) while grown into nanoscale. For example, under suitable growth conditions, SnTe nanocubes could be synthesized due to the face-centered cubic crystal structure with six lowest energy crystalline faces of (100). ${ }^{[31]}$ Consequently, the thermodynamic equilibrium state must be broken, and the controllability of kinetics must be artificially introduced to stimulate the 2D anisotropic growth of non-layered materials. Up to now, both bottom-up method and the strategy combining bottomup self-assembly with top-down method have been developed to synthesize 2D non-layered materials. Figure 1a shows the schematic diagram of the bottom-up synthesis using van der Waals epitaxy (vdWE) as an example. ${ }^{[37]}$ Similar with chemical vapor deposition (CVD) method, sources (like atoms and molecules) firstly adsorb/de-adsorb onto/from the substrates. Then the adatoms migrate on the surfaces and nucleate at the favorable places, like defects, steps and edges. After that, the 2D structures will form through the following two ways: the nuclei grow up directly along the lateral directions (up process in Figure 1a); or the preformed small nanocrystals meet each other and coalesce for the demand of the lowest system energy (bottom process in Figure 1a). No matter which process it follows, two conditions need to be met: 1) 2D anisotropic growth is preferred through controlling the growth parameters, such as temperatures, pressures and gas flow rates; 2) adatoms/ small nanocrystals are able to migrate/twist on the substrate surfaces. ${ }^{[30]}$ These requirements can be met by using dangling bond free substrates, like mica and 2D layered materials. Hence, vdWE has become one of the most important synthesis methods to grow non-layered materials with 2D geometry. ${ }^{[33,37]}$ Besides vdWE, two other bottom-up strategies have also been developed, including template-directed synthesis method ${ }^{[34,36]}$ and surfactant-assisted self-assembly way. ${ }^{[20,22]}$ For the former, 2D templates (2D layered materials in the most cases) are synthesized firstly, on the basis of which 2D non-layered materials are obtained through direct growth on the top of the template surfaces ${ }^{[29,38]}$ or through phase transition process. ${ }^{[34,36]}$ For the latter, 2D non-layered materials are obtained through laterally self-assembling preformed nanocrystals whose top and bottom surfaces have been passivated with surfactants. ${ }^{[22]}$ On the other hand, growth strategy that is combined with bottom-up and topdown methods has also been developed. ${ }^{[2,27]}$ Figure $1 \mathrm{~b}$ shows the corresponding schematic diagram. Using organic surfactants as constraint layer, non-layered materials can be grown only in the confined 2D area, hence, forming the bulk materials with lamellar non-layered materials and organic spacers stacking alternately. Due to the weak interaction strength between the organic spacers and the inorganic lamellas, 2D non-layered materials can be collected by peeling off the asgrown bulk materials and removing the organics. In brief, all the methods share the same mechanism: external intervention must be introduced to kinetically promote the 2D anisotropic growth. In the following parts, we will give a detailed review of the synthesis methods that have been developed for 2D nonlayered materials.

\subsection{Synthesis Methods}

So far, various methods have been developed to prepare 2D materials, such as mechanical exfoliation, ion-intercalation, 
and chemical vapor deposition, wet-chemical synthesis, and so on. ${ }^{[7]}$ Among them, chemical vapor deposition and wet-chemical methods are usually utilized to synthesize 2D non-layered materials. We will focus on these two methods in the following part.

\subsubsection{Dry Methods}

CVD method has shown great superiority in preparing 2D nanomaterials with high crystal quality, high yield, and large scale. However, because of the difficulties of anisotropic growth, general CVD approach cannot meet the requirements for synthesizing 2D non-layered crystals. Recently, this obstacle has been overcome by introducing suitable epitaxial substrates with chemically inert and smooth surfaces into the growth process. And the so-called van der Waals epitaxial (vdWE) growth has exhibited great ability to realize highly anisotropic growth for 2D non-layered materials. Different from the conventional epitaxial growth, such as metal organic chemical vapor deposition (MOCVD) and molecular beam epitaxy (MBE), vdWE does not require strict requirement of lattice matching between the growing layer and the substrates because: (1) the vdWE substrates can act as the thermal mismatch buffer layer to relax the thermal stress, (2) the migration of the reaction precursors on the substrates can be further facilitated as a result of the relatively weak van der Waals interaction at the interfaces during epitaxial growth, thus resulting in highly anisotropic growth with relatively large lateral sizes, high crystal quality, and even defect-free crystals. ${ }^{[30,32,37]}$

Usually, mica, $h$-BN, graphene and $\mathrm{MoS}_{2}$ etc. can be used as the vdWE substrates because of their atomically smooth and chemically inert surfaces. For example, our group has presented a general strategy to prepare 2D non-layered materials, including $\mathrm{Pb}_{1-x} \mathrm{Sn}_{x} \mathrm{Se}, \mathrm{SnTe}, \mathrm{Te}$, and $\mathrm{PbS}$ nanoplates via vdWE..$^{[9,32,37,39]}$ We chose hexagonal Te as the first attempt in which fluorophlogopite mica was used as the growth substrates ${ }^{[37]}$ Figure 2a gives optical microscope images of the Te hexagonal nanoplates with lateral dimension of about $6-10 \mu \mathrm{m}$. Atomic force microscope (AFM) was carried out to identify the thickness of the Te nanoplates, as shown in Figure 2b. The average lateral dimension was $4.5 \mu \mathrm{m}$ with thickness of 30-60 $\mathrm{nm}$. When the thickness increased to $70-90 \mathrm{~nm}$, the average lateral dimension increased to $6.1 \mu \mathrm{m}$. Further, as ternary compound with more complex structure, ultrathin $\mathrm{Pb}_{1-x} \mathrm{Sn}_{x} \mathrm{Se}$ nanoplates were synthesized also on mica. ${ }^{[32]}$ The thickness of the $\mathrm{Pb}_{1-x} \mathrm{Sn}_{x} \mathrm{Se}$ nanoplates distributed mainly in 15-45 nm, shown in Figure 2c. It was found that the growth mechanism follows the Volmer-Weber (VW) model. The growth process could be divided into two major steps: (1) atoms firstly gather into individual nanoislands, and (2) the as-obtained nanoislands coalesce and gradually grow into a regular nanoplate. Since the lateral (100) equivalent planes facets are more active than the top (001) surface for Te nanoplates, and (110) equivalent surfaces of the $\mathrm{Pb}_{1-x} \mathrm{Sn}_{x} \mathrm{Se}$ nanoplates have higher activation than the other facets, combining the effects from vdWE substrates, the vertical growth was largely suppressed and highly anisotropic 2D growth of non-layered $\mathrm{Te}$ and $\mathrm{Pb}_{1-x} \mathrm{Sn}_{x} \mathrm{Se}$ can be successfully realized. Benefiting from the planar geometry and narrow band gaps, these two materials are ideal candidates for flexible infrared photodetection. Figure 2d indicates the photograph of $2 \mathrm{D} \mathrm{Pb}_{1-x} \mathrm{Sn}_{x} \mathrm{Se}$ flexible optoelectronic devices. The $\mathrm{Pb}_{1-\mathrm{x}} \mathrm{Sn}_{\mathrm{x}} \mathrm{Se}$ nanoplates exhibited reversible, stable photoresponse and broad spectra detection $(375,473$, 632, 800, and $980 \mathrm{~nm}$ ). Notably, Te shows good stability in photoresponse even after bending for 100 times.

In order to verify the universality of the vdWE methods for 2D non-layered growth, our group further synthesized 2D $\mathrm{Pb}_{1-x} \mathrm{Sn}_{x} \mathrm{Se}$ on $h$-BN thermal/lattice mismatch buffer layer for the integration with $\mathrm{Si}$ substrates. ${ }^{[33]} h$-BN is an appealing dielectric substrate, free of dangling bonds and surface charge traps, and with atomically planar surface. It is an efficient buffer layer for damping the scattering from the substrate surface roughness, charged surface states and impurities, and $\mathrm{SiO}_{2}$ surface optical phonons. In addition, $h$-BN exhibits high compatibility with flexible and $\mathrm{SiO}_{2} / \mathrm{Si}$ substrates. Figure $2 \mathrm{e}$ shows the optical microscope image indicating the fact that $\mathrm{Pb}_{1-x} \mathrm{Sn}_{x} \mathrm{Se}$ nanoplates can be grown on amorphous $\mathrm{SiO}_{2} / \mathrm{Si}$ substrates through a few-layer $h$-BN, which further confirms vdWE is an efficient method to grow 2D non-layered materials.

Because general vdWE substrates own smooth, dangling bond-free surfaces, such as graphene, $h$ - $\mathrm{BN}$, it is a key point to control the nucleation at the initial stage of growth. Wang et al. found $\mathrm{PbS}$ nanoplates prefer to grow on the edge of graphene and develop an edge-contact growth method to construct $\mathrm{PbS}$ nanoplate-graphene heterostructures, as shown in Figure 3a. ${ }^{[40]}$ This is because the unsaturated carbon atoms at the edge of graphene provide higher nucleation rates. Combining density functional theory, they found $\mathrm{PbS}$ nanoplates are covalently bonded to graphene edge with strong chemical hybridization. Comparison between top-contact model (vdW model as shown in Figure 3b) and zig-S model (Figure 3c) shows that the charge redistribution chiefly occurs at the contact region and the charge density increases in the bonding region.

In order to artificially enhance the nucleation rates, Wen et al. modified the vdWE surfaces (mica) by introducing $\mathrm{NaCl}$ using a polydimethylsiloxane (PDMS) stamp. ${ }^{[39]}$ Combining photolithography and PDMS stamp technique, they developed a method to fabricate patterned PbS-nanoplates arrays, as shown in Figure 3d. The morphology of the PbS nanoplates array was investigated by SEM under different reaction time, as shown in Figure 3e-g. First, a large amount of nucleus distributes evenly in the entire growth substrate within the reaction time of $30 \mathrm{~s}$. Then, plenty of irregular nanoplatelets form and tend to coalesce with each other, when increasing the reaction time to $1 \mathrm{~min}$. Finally, when the reaction time extends to $2 \mathrm{~min}$, regular square nanoplates appear exposed with four equivalent (200) crystal surfaces.

Besides using 2D layered materials as buffer layers, another strategy to grow 2D non-layered materials directly on $\mathrm{SiO}_{2} / \mathrm{Si}$ wafers are lifting the nanomaterials up of the substrate surfaces. Figure $2 \mathrm{f}$ shows the schematic diagram of the vertical $\mathrm{Pb}_{1-x} \mathrm{Sn}_{x}$ Te nanoplates synthesized on $\mathrm{SiO}_{2} / \mathrm{Si}$ substrate. ${ }^{[9]}$ The thickness of the nanoplates mainly distributed in $40 \mathrm{~nm}$ with lateral size as large as $20 \mu \mathrm{m}$. It was found that the $\mathrm{Pb}_{1-x} \mathrm{Sn}_{x} \mathrm{Te}$ nanoplates form through the following anisotropic growth mechanism: the nucleus grow and become stable firstly because of the strong chemical interaction between the adatoms and 

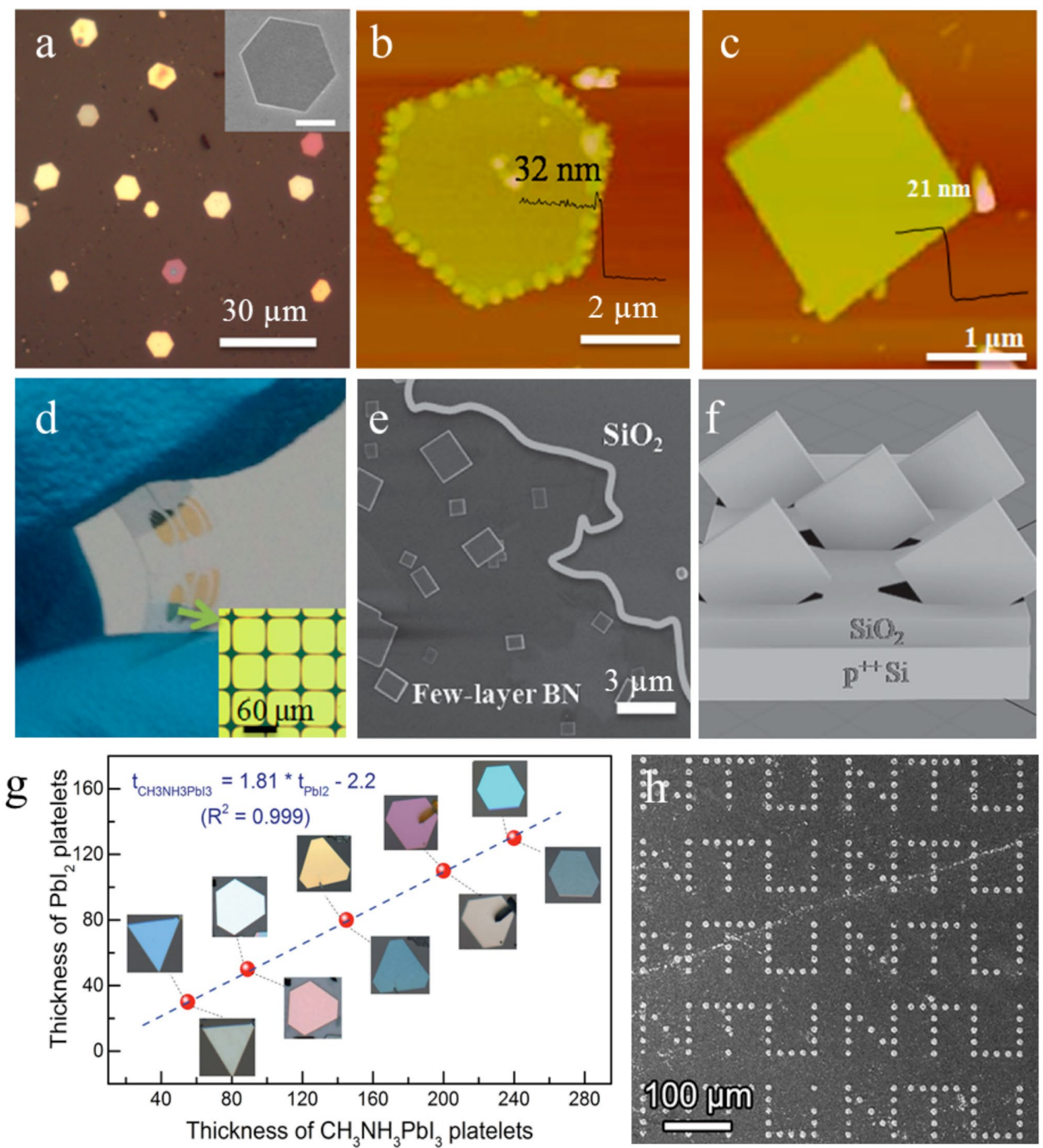

Figure 2. a) Optical microscopy and b) AFM image of the Te hexagonal nanoplates. Inset of (a) shows the corresponding SEM image with scale bar $=4 \mu \mathrm{m}$. Reproduced with permission. ${ }^{[37]}$ Copyright 2014, American Chemical Society. c) AFM image of the $\mathrm{Pb}_{1-x} \mathrm{Sn}_{x} \mathrm{Se}$ nanoplate with thickness of $21 \mathrm{~nm}$. d) Photograph of the 2D Pb${ }_{1-x} \mathrm{Sn}_{x}$ Se flexible optoelectronic devices; inset is the OM image of electrodes pattern. Reproduced with permission. ${ }^{[32]}$ Copyright 2015, American Chemical Society. e) $\mathrm{Pb}_{1-x} \mathrm{Sn}_{x}$ Se nanoplates grown on h-BN. Reproduced with permission. ${ }^{[33]}$ Copyright 2015, Wiley. f) Schematic illumination of the vertically oriented $\mathrm{Pb}_{1-x} \mathrm{Sn}_{x}$ Te nanoplates on $\mathrm{SiO}_{2} / \mathrm{Si}$ substrates. Reproduced with permission. ${ }^{[9]} \mathrm{Copyright} 2016, \mathrm{Wiley}$. g) Thickness of the $\mathrm{Pbl}_{2}$ platelets before (images above data line) and after being converted to Perovskite (images below data line). Reproduced with permission. ${ }^{[34]}$ Copyright 2015, Wiley. h) SEM image of the perovskite/h-BN "NTU" arrays. Reproduced with permission. ${ }^{[36]}$ Copyright 2015 , Wiley.

the dangling bonds of $\mathrm{SiO}_{2}$. Since the highly disordered surface states of the $\mathrm{SiO}_{2}$, the lateral growth is suppressed and $\mathrm{Pb}_{1-x} \mathrm{Sn}_{x} \mathrm{Te}$ nanoplates grow vertically in order to minimize the total system energy. Therefore, the nucleus continues to grow along the vertical direction instead of the surface of $\mathrm{SiO}_{2} / \mathrm{Si}$.

All the strategies mentioned above are about synthesizing 2D non-layered materials directly through CVD-based methods. Another way is to firstly grow 2D layered materials "precursors" followed by a conversion process to obtain the corresponding 2D non-layered materials. ${ }^{[34-36,41]}$ Comparing with the strict requirements of growth parameters of vdWE and vertical growth methods, this two-step strategy is relatively facile to control. However, it works only on the specific material species, such as organic-inorganic perovskites of $\mathrm{RNH}_{3} \mathrm{PbX}_{3}$. Xiong et al. demonstrated the synthesis of $2 \mathrm{D}$ lead halide perovskite family $\mathrm{CH}_{3} \mathrm{NH}_{3} \mathrm{PbX}_{3}(\mathrm{X}=\mathrm{Cl}, \mathrm{Br}, \mathrm{I})$ by converting 2D layered $\mathrm{PbX}_{2}{ }^{[34]}$ Nanoplatelets with lateral dimensions of $5-30 \mu \mathrm{m}$ and thicknesses from several atomic layers to several hundred nanometers were grown through two steps: first, lead halide nanoplatelets $\left(\mathrm{PbCl}_{2}, \mathrm{PbBr}_{2}\right.$, and $\left.\mathrm{PbI}_{2}\right)$ were grown on muscovite mica substrates by van der Waals epitaxy. Due to the layered structure of the lead halide, few layer even monolayer nanoplates could be obtained. Secondly, lead halide nanoplatelets could be converted to perovskites simply via a gas-solid hetero-phase reaction. The thickness of the $\mathrm{CNH}_{3} \mathrm{PbI}_{3}$ perovskite platelets are about 1.81 times thicker compared to the pristine $\mathrm{PbI}_{2}$, which agrees with the lattice constant ratio of the two compounds along the $\mathrm{c}$ axis, as shown in Figure $2 \mathrm{~g}$. This method proposes a novel strategy to control the thickness of non-layered perovskites by monitoring the thickness of 
www.MaterialsViews.com
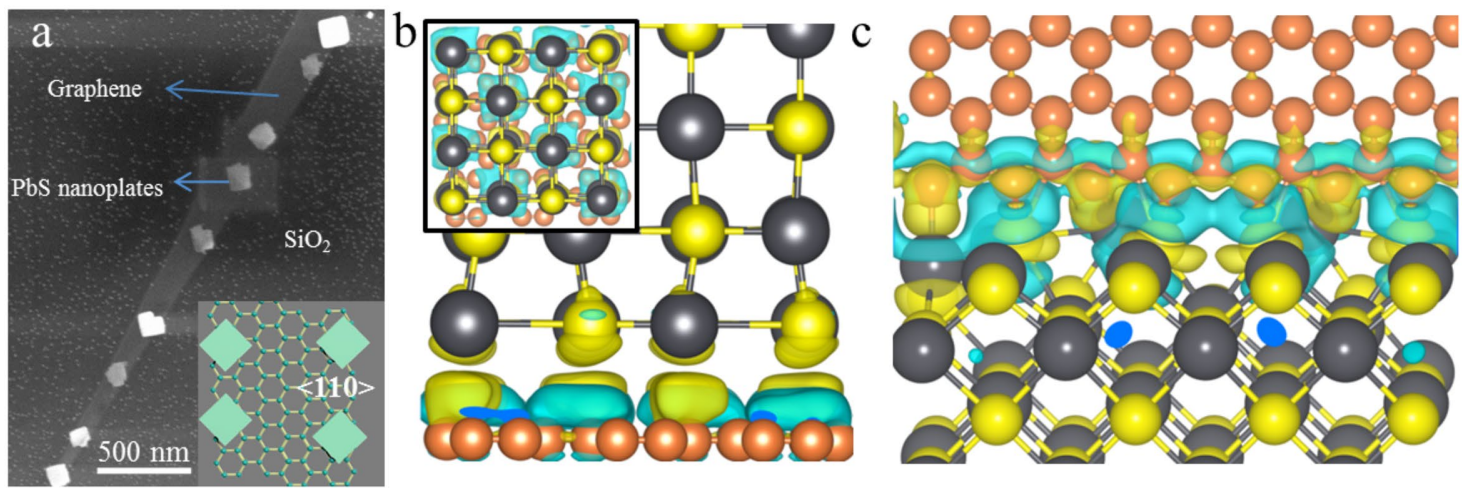

d
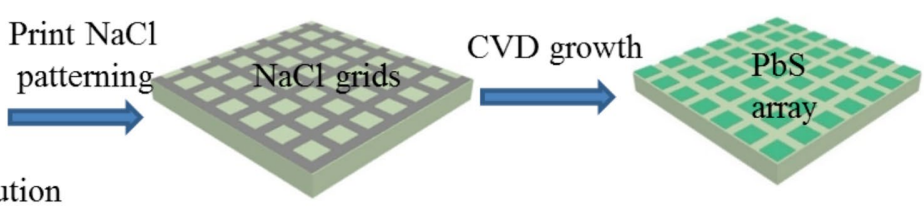

$\mathrm{NaCl}$ aqueous solution
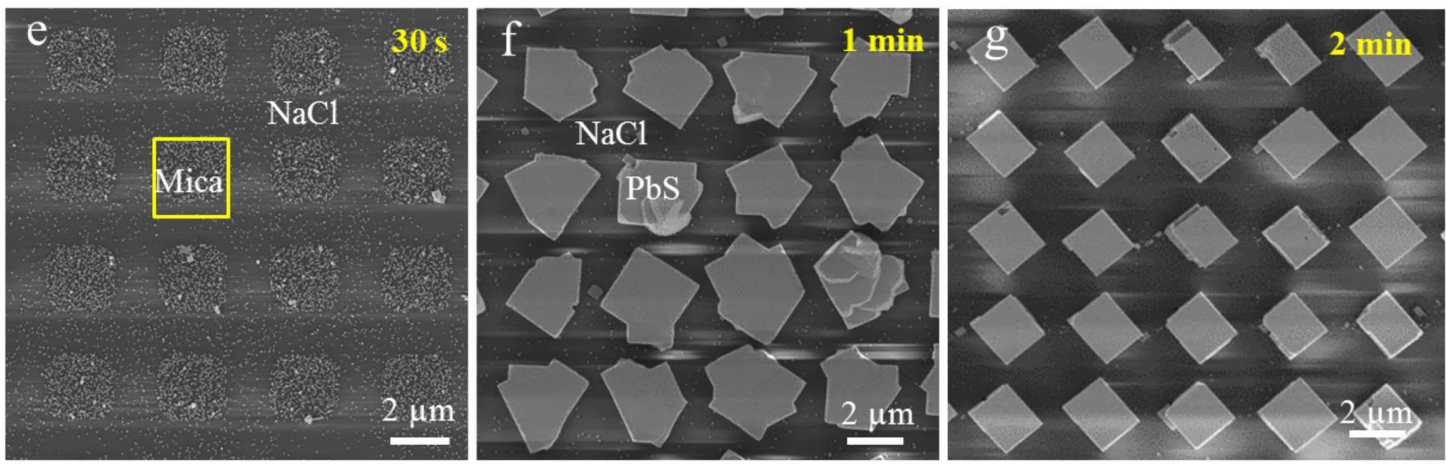

Figure 3. a) SEM image of the epitaxial PbS nanoplates heterostructure. PbS nanoplates contact with graphene edges along their $<110>$ directions, as shown in the inset. b) Charge density difference in the hybrid PbS nanoplates-graphene heterostructure. The yellow and blue regions represent charge accumulation and depletion, respectively. The red ball is C, yellow ball is S; and the black ball is Pb. Inset shows the charge distribution in bottom (100) surface of PbS nanoplates. c) Charge density deference in the zig-S model. (a-c) Reproduced with permission. ${ }^{40]}$ Copyright 2016 , Wiley. d) Schematic representation of procedures for patterning of 2D PbS nanoplates arrays. The SEM images of the PbS nanoplates arrays under different reaction time e) $30 \mathrm{~s}$, f) $1 \mathrm{~min}$, and g) $2 \mathrm{~min}$. (d-f) Reproduced with permission. ${ }^{[39]}$ Copyright 2016, Wiley.

2D-templates. Taking advantage of a similar strategy, Liu et al. reported the growth of perovskite arrays on graphene, h-BN and $\mathrm{MoS}_{2}$ substrates. ${ }^{[36]}$ Figure $2 \mathrm{~h}$ exhibits the SEM image of the perovskite/ $h$-BN “NTU” arrays. Recently, Duan et al. demonstrated the wafer-scale synthesis of perovskite crystals arrays through controlling the periodical hydrophobic/hydrophilic regions by a monolayer hydrophobic (octadecyl)trichlorosilane (OTS). ${ }^{[41]} \mathrm{PbI}_{2}$ nanoplates were selectively grown on patterned hydrophilic area. Thereafter, perovskite crystals were achieved by a following gas-solid hetero-phase reaction.

\subsubsection{Wet Chemistry Methods}

Wet chemistry method has turn to be a popular way to obtain the most kinds of 2D non-layered materials due to its low-cost, high-output and controllability. 2D templates and surfactant agents are often used in this method to ensure non-layered materials growing into a two-dimensional structure. According to the different mechanism, wet chemistry method can be classified as the followings: surface-energy-controlled synthesis (SECS), template-directed synthesis (TDS), confined space synthesis (CSS), colloidal synthesis (CS) and other solvothermal methods.

Metals such as $\mathrm{Ag},{ }^{[42]} \mathrm{Pd},{ }^{[11,43]} \mathrm{Rh},{ }^{[13,44]}$ and $\mathrm{Ru}{ }^{[45]}$ can be synthesized by surface-energy-controlled synthesis (SECS). Poly (vinylpyrrolidone) (PVP) ${ }^{[13,42,45]}$ and cetyltrimethylammonium bromide $(\mathrm{CTAB})^{[11]}$ are usually utilized as surfactant agents to attach to the low index surfaces of non-layered materials in order to reduce the growth rate of these directions and promote the 2D growth. As a typical example, Ag nanoplates with high aspect ratio was reported by $\mathrm{Q}$. Zhang and co-workers, in which PVP and citrate ion work as surfactant agents. ${ }^{[42]}$ The schematic illustration is shown in Figure 4a. Firstly, PVP together with citrate ion induce the high output of plate-like seeds with two (111) twins. After removing PVP, citrate ligands on (111) facets confined the growth along the vertical axis and promoted the $2 \mathrm{D}$ growth. In addition, some reports have also indicated that 
a

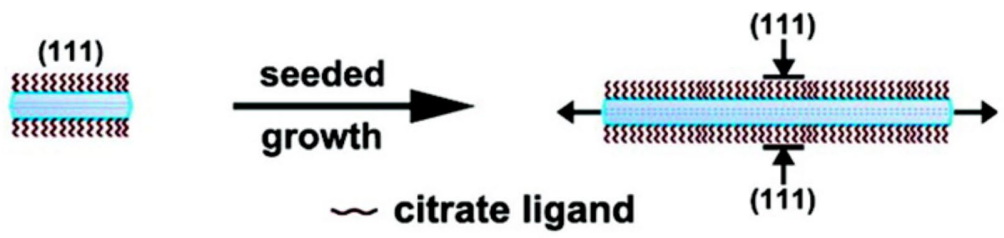

$\mathrm{b}$

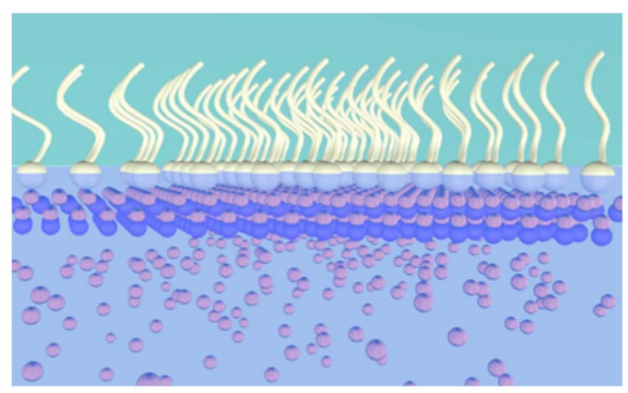

c

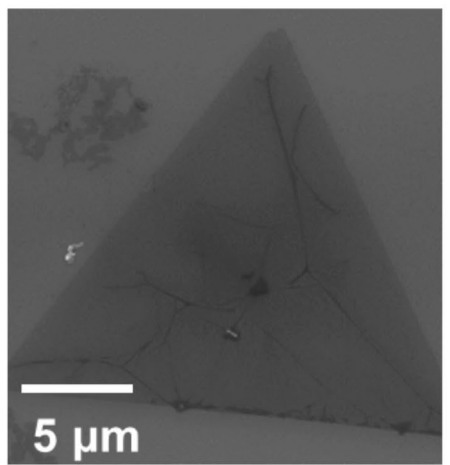

1-Amino-9-octadecene
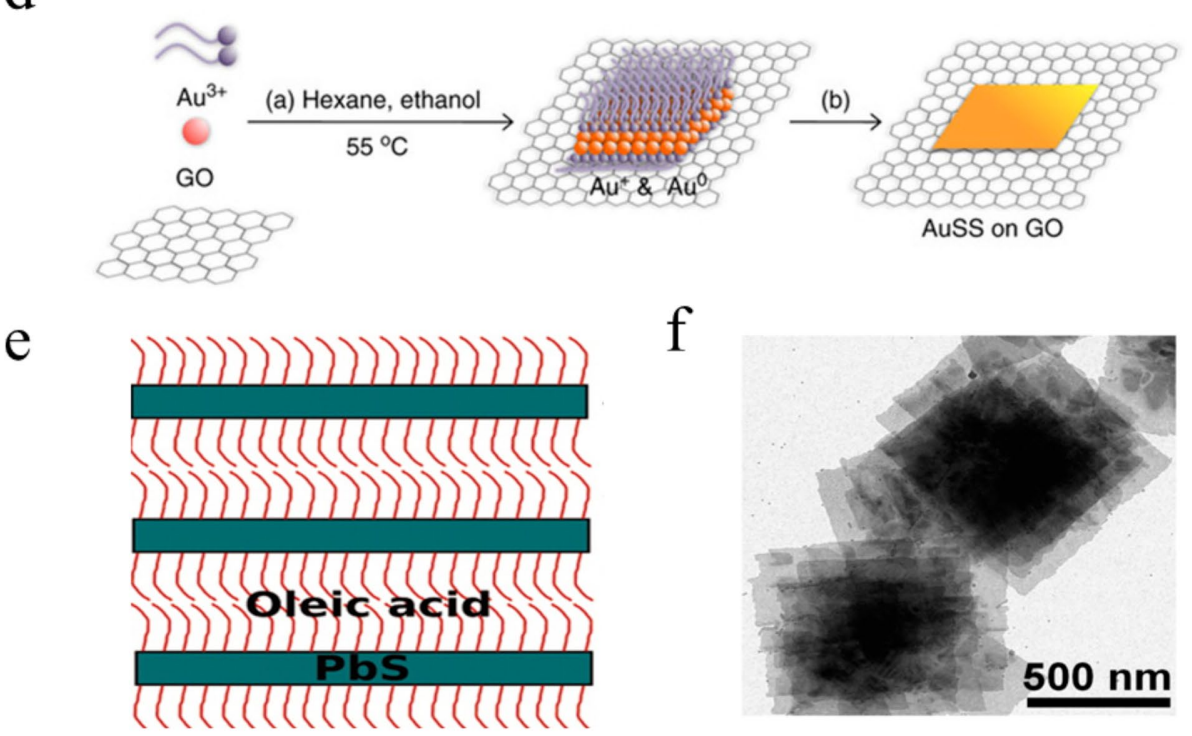

Figure 4. Schematic illustration of the example process of surface-energy-controlled synthesis (SECS), template-directed synthesis (TDS), confined space synthesis (CSS) and colloidal templated synthesis (CTS). a) Schematic illustration of surface-energy-controlled synthesis of Ag nanoplates. Reproduced with permission. ${ }^{[42]}$ Copyright 2010, American Chemical Society. b) Schematic illustration of the synthesis of ZnO nanosheets directed by surfactant monolayer. c) SEM image of a typical ZnO nanosheet. (b and c) Reproduced with permission. ${ }^{[47]}$ Copyright 2016, Nature Publishing Group. d) Schematic illustration of the formation mechanism of Au nanoplates using GO as templates. Reproduced with permission.$^{[12]}$ Copyright 2011 , Nature Publishing Group. e) Schematic illustration of the synthesis of ultrathin single-crystal PbS sheets using OA as colloidal templates. $\mathrm{f}$ ) TEM image of the obatined ultrathin single-crystal PbS sheets. (e and f) Reproduced with permission. ${ }^{[22]}$ Copyright 2010, AAAS.

CO can also work as the shape controller to obtain ultrathin metal nanosheets. ${ }^{[11,43,44,46]}$ In the growth process, CO prefers to be a ligand to the selected facets of $\mathrm{Pd}$ and $\mathrm{Rh}$, through which 2D anisotropic growth of metals was facilitated.

Confined space synthesis (CSS) provides a 2D space so that the specific reaction is constrained in. To minimize their surface energy, the reactive precursor ions in this space have to array in a crystallographic face and then for the wanted 2D nonlayered nanosheets. For example, through this method, Xudong Wang's group fabricated single-crystalline $\mathrm{ZnO}$ nanosheets with large lateral sizes for the first time at the water-air interface as shown in Figure 4b. ${ }^{[4]}$ By introducing high-concentration sodium dodecyl sulfate, the water-air interface was built up between air and the solution, in which the $\mathrm{ZnO}$ growth was only limited. In addition, the sodium dodecyl sulfate also works as surfactant agents to guide the growth of $\mathrm{ZnO}$ nanosheets.

Different with the confined growth method, templatedirected synthesis (TDS) offers 2D templates to help the 2D growth. Graphene oxide (GO) has long been used as the 2D template to direct the growth of various non-layered materials such as $\mathrm{Au},{ }^{[12,15,16]} \mathrm{SnO}_{2},{ }^{[48]}$ and $\mathrm{Al}_{2} \mathrm{O}_{3} .{ }^{[49]}$ Schematic illustration of the formation process of $\mathrm{Au}$ nanosheets using $\mathrm{GO}$ as templates is shown in Figure 4d. Interestingly, after growth with GO as 2D template, Au nanosheets were used as template to 
grow Au@Ag, ${ }^{[16]} \mathrm{Au@Pt,} \mathrm{and} \mathrm{Au@Pd}{ }^{[15]}$ core-shell nanosheets. $\mathrm{Li}$ and co-workers developed a bottom-up method to synthesize ultrathin $2 \mathrm{D} \mathrm{Al}_{2} \mathrm{O}_{3}$ nanosheets with $\mathrm{GO}$ as template. ${ }^{49]}$ Using homogeneous deposition method, a layer of amorphous aluminum hydroxide precursors was formed continuously on the GO template. After calcination at $800{ }^{\circ} \mathrm{C}$ in atmosphere, the as-formed aluminum hydroxide layer was converted into $\mathrm{Al}_{2} \mathrm{O}_{3}$ nanosheets perfectly duplicating the morphology of GO template. Remarkably, the authors found that this strategy is feasible only for synthesizing metal oxides with amorphous precursors, including $\mathrm{Al}_{2} \mathrm{O}_{3}$ and $\mathrm{ZrO}_{2}$. However for the other metal oxides like $\mathrm{Mn}_{2} \mathrm{O}_{3}, \mathrm{Fe}_{2} \mathrm{O}_{3}$, and $\mathrm{ZnO}$, porous particles instead of 2D nanosheets were synthesized because their precursors were crystals which were not continuously covered on the GO templates. Zhu and co-workers obtained non-layered $\mathrm{NiO}$ nanosheets with high specific capacitance through direct transformation from layered a- $\mathrm{Ni}(\mathrm{OH})_{2}$ templates. ${ }^{[50]}$ A similar method has also been studied for the synthesis of $\mathrm{Co}_{3} \mathrm{O}_{4}$ nanosheets from a-Co(OH) $)_{2} \cdot{ }^{[51]}$ Colloidal templated synthesis (CTS) is also a promising way to prepare 2D non-layered materials by oil template phase transformation. Different from TDS, acid (OA), Octylamine (OTA) and oleylamine (OM) surfactants ${ }^{[1,22,23,28,29,52-54]}$ perform as colloidal templates for guiding the crystal growth. With the long-chain ligands based on oleyl amine or oleic acid, precursor can be only assembled along one face. After oriented assembly, organic shells are removed and 2D materials are left. Horst Weller and coworkers used $\mathrm{OA}$ to synthesis ultrathin single-crystal $\mathrm{PbS}$ sheets with dimensions on the micrometer scale for the first time as shown in Figure 4e. ${ }^{[22]}$ They found that OA ligands can selectively attach on the $(100)$ facets of PbS leading to the confinement of structures on the vertical directions. Sometimes, layered precursors with ligands need further reaction to fabricate objective materials. Hua Zhang's group obtained ultrathin $\mathrm{CuS}$ nanosheets after treating layered $\mathrm{CuCl}(\mathrm{OTA}, \mathrm{OM})_{x}$ with $\mathrm{S}$ powder at $95^{\circ} \mathrm{C} .{ }^{[23]}$ Similarly, ultrathin CdSe nanosheets were synthesized by treating $\mathrm{CdCl}_{2}(\mathrm{OA})_{2}$ with Se powder. ${ }^{[28]}$

Some other solvothermal methods have also been used to obtain 2D non-layered materials such as $\mathrm{SnO}_{2},{ }^{[55]} \mathrm{Mn}_{3} \mathrm{O}_{4},{ }^{[56]}$ and $\mathrm{Co}_{9} \mathrm{Se}_{8}{ }^{[57]}$ Compared with dry method, wet chemistry method occur in solution and can be used for synthesizing more types of 2D non-layered materials, such as metal, metal oxide, metal chalcogenides. Generally, wet chemistry method plays an important role in 2D non-layered material synthesis.

\section{Applications}

\subsection{Catalysis}

\subsection{Catalysis of Water Splitting}

Photocatalytic water splitting gives rise to the green hydrogen hope of powering our planet without any contribution to global warming. ${ }^{[58]}$ In a typical conversion of solar energy to chemical energy, a flux of photon is first absorbed by the designed semiconductor and produce high energy charge carriers. The generated charge carriers then separate from each other and diffuse to catalytically active sites at the semiconductor/liquid interfaces to carry out the water photocatalysis. Charge separation is the crucial step to realize the overall water splitting as the energetic holes/electrons drive the oxygen/hydrogen-evolution half reactions. ${ }^{[59]}$ Following the pioneering work done by Fujishima and Honda in 1972,[60] which demonstrated the electrochemical photolysis of water splitting on $\mathrm{TiO}_{2}$ semiconductor, various other materials have been developed for photocatalysis of water splitting. In order to harvest sufficient energy from the sun, the design of visible light driven photocatalysts is greatly sought by virtue of considering the thermodynamic favorability of the valance and conduction band positions. Nonlayered 2D materials are promising candidates to drive such photocatalysis owing to their unique properties, like the large amount of exposed active atoms with unsaturated coordination that are not observed in their bulk counterparts. In this family, free standing ZnSe single layer with four-atomic thickness has been reported by Xie's group. ${ }^{[26]}$ Using n-propylamine, they made a lamellar hybrid intermediate of bulk $\mathrm{Zn}_{2} \mathrm{Se}_{2}$, followed by exfoliation and organic species removal to produce the final product of ultra-flexible ZnSe single layer. Compared with bulk ZnSe, these 2D ZnSe layers are endowed with a unique electronic structure and an excellent structural stability which are important characteristics for enhanced photoelectrochemical (PEC) solar water splitting (Figure 5a). It was found that the freestanding ZnSe single layers exhibited a photocurrent density of $2.14 \mathrm{~mA} \mathrm{~cm}^{-2}$ at $0.72 \mathrm{~V}$ under a irradiation by a $300 \mathrm{~W}$ Xe lamp, which is 195 times higher than that of its bulk counterpart (Figure 5b). What's more, the incident photon-to-current conversion efficiency (IPCE) of $42.5 \%$ is remarkably higher than the investigated $\mathrm{ZnSe}(0.25 \%), \mathrm{Zn}_{2} \mathrm{Se}_{2} / \mathrm{n}$-propylamine (0.4\%), ZnSe/n-propylamine octuplet layers (4.3\%), ZnSe/npropylamine quadruple layers (5\%) and ZnSe/n-propylamine double layers (12.1\%). (Figure 5c) Similarly Xu et al., ${ }^{[61]}$ reported $\mathrm{CdS}$ nanosheets with a thickness of $4 \mathrm{~nm}$ as visible-light-driven water splitting photocatalysts for hydrogen generation. They used ultrasonication-induced aqueous exfoliation approach by utilizing inorganic-organic lamellar hybrid of CdS-DETA, water and L-cysteine as starting material, dispersion medium and stabilizing agent, respectively. The obtained ultrathin nanosheets exhibited photocatalytic hydrogen production rate of $41.1 \mathrm{mmol}$ $\mathrm{h}^{-1} \mathrm{~g}^{-1}$ in the presence of sacrificial agent, which is close to 5.5 times and 6.1 times faster than that of CdS-DETA hybrid nanosheets and CdS nanosheet-based aggregates, respectively (Figure $5 \mathrm{e}$ and $\mathrm{f}$ ). In addition, no noticeable decrease was detected even in a $12 \mathrm{~h}$ test. Very recently, our group reported Se enriched surface of $\mathrm{NiSe}_{2}$ to accelerate the charge transfer during the HER process. ${ }^{[62]}$ The surface modification on such 2D non-layered material enhanced the catalytic capacity toward hydrogen generation as compared to the stoichiometric $\mathrm{NiSe}_{2}$ nanosheet. Experimental and theoretical works made by Xie's group manifested the active role of oxygen vacancy in ultrathin porous $\mathrm{In}_{2} \mathrm{O}_{3}$ nanosheet that brought a visible light active 2D non-layered photocatalyst for water splitting. ${ }^{[19]}$ Fast-heating of cubic $\operatorname{In}(\mathrm{OH})_{3}$ sheets was performed to realize the fabrication of 5-atom-thick $\mathrm{In}_{2} \mathrm{O}_{3}$ porous sheets with O-vacancies featuring a narrowed band gap and higher carrier concentration. The appearance of new defect level in the oxygen-vacancy-rich $\operatorname{In}_{2} \mathrm{O}_{3}$ initiated the increased visible light absorption owing to the electron transition from the valence band to the new defect level 
a

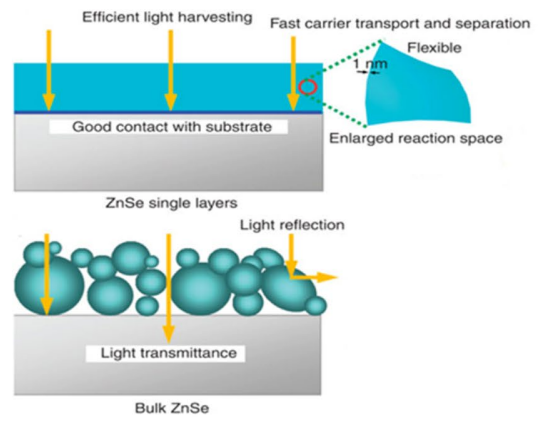

d
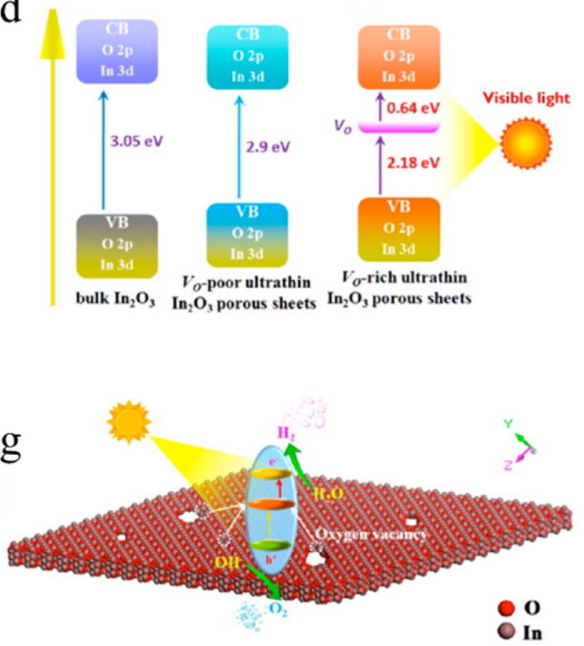

b

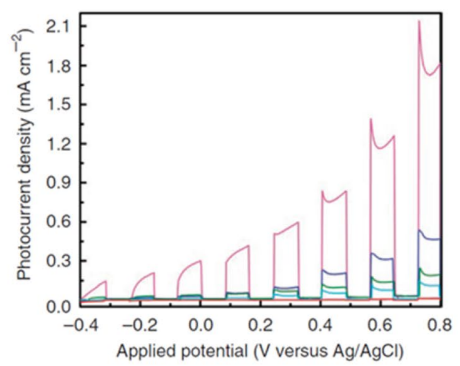

e

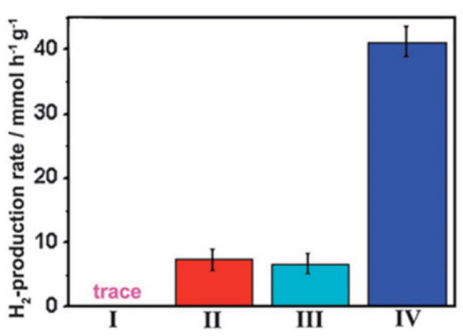

$\mathrm{h}$

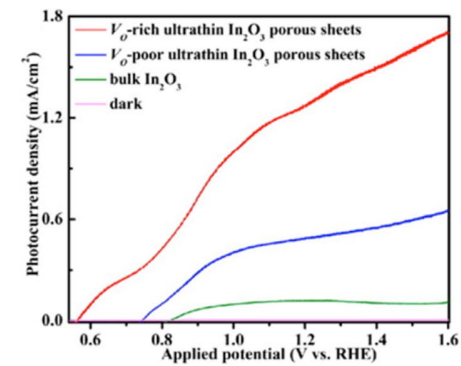

$\mathrm{C}$

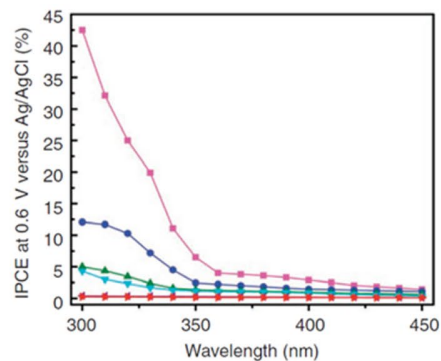

$\mathrm{f}$

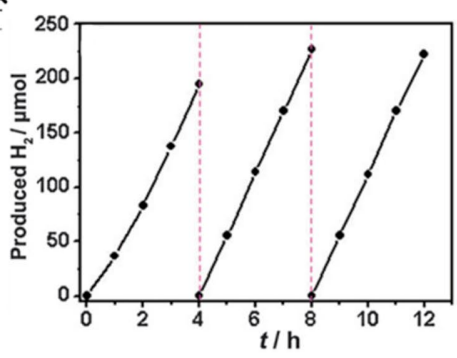

i

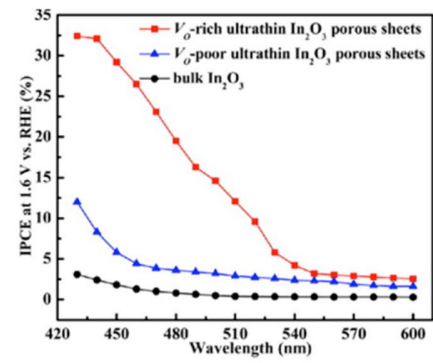

Figure 5. a) Advantages of using large-area flexible ZnSe freestanding single layers versus bulk ZnSe deposited on indium tin oxide (ITO)-coated glass as the photoelectrodes for solar water splitting. b) Photocurrent density versus applied potential curves under chopped $300 \mathrm{~W} X \mathrm{Xe}$ lamp irradiation; and c) IPCE of ZnSe single layers (magenta), ZnSe- n-propylamine double layers (blue) and ZnSe-n-propylamine quadruple layers (olive), ZnSe-npropylamine octuple layers (cyan), $\mathrm{Zn}_{2} \mathrm{Se}_{2}$-n-propylamine (purple) and bulk $\mathrm{ZnSe}$ (red). (a-c) Reproduced with permission. ${ }^{[26]}$ Copyright $2012, \mathrm{Nature}$ Publishing Group. d) Schematic band structure revealing the appearance of new defect level in vacant rich $\ln _{2} \mathrm{O}_{3}$ nanosheet Reproduced with permission. ${ }^{[19]}$ Copyright 2014, American Chemical Society. e) Comparison of the photocatalytic hydrogen evolution reaction activity of CdS nanoparticles (I), CdS-DETA nanosheets (II), CdS nanosheet-based aggregates (III) and ultrathin CdS nanosheet dispersion (IV) f) Time course of photocatalytic $\mathrm{H}_{2}$ production over ultrathin CdS nanosheets. (e and f) Reproduced with permission. ${ }^{[61]}$ Copyright 2013, Royal Society of Chemistry. g) Schematic solar water splitting of vacant-rich ultrathin $\mathrm{In}_{2} \mathrm{O}_{3}$ nanosheets. h) Photocurrent vs applied potential curves under $300 \mathrm{~W}$ Xe lamp irradiation $(\lambda>420 \mathrm{~nm}$ ). i) The corresponding IPCE at $1.6 \mathrm{~V}$ vs RHE of vacant rich ultrathin $\ln _{2} \mathrm{O}_{3}$ nanosheets, vacant poor ultrathin $\ln _{2} \mathrm{O}_{3}$ nanosheets and the bulk $\mathrm{In}_{2} \mathrm{O}_{3}$. (g-i) Reproduced with permission. ${ }^{[19]}$ Copyright 2014, American Chemical Society.

or from the defect level to the conduction band (Figure $5 \mathrm{~d}$ and g). Figure $5 \mathrm{~h}$ and i depict the superior performance of $\mathrm{In}_{2} \mathrm{O}_{3}$ porous sheets with rich oxygen vacancy as compared to the one with poor oxygen vacancy and the bulk.

\subsubsection{Catalysis of CO Conversion and Organic Reactions}

Another application of 2D non-layered materials is catalytic oxidation of carbon monoxide, which is very toxic to human and animals. This was demonstrated on pits confined ultrathin $\mathrm{CeO}_{2}$ nanosheets ${ }^{[21]}$ (Figure 6a) and $0.6 \mathrm{~nm}$ thick $\mathrm{SnO}_{2}$ nanosheets, ${ }^{[63]}$ as reported by Xie's group. In $\mathrm{CeO}_{2}, 20 \%$ pit occupancy was modeled in view of maximizing the number of coordinately unsaturated active sites. Temperature dependent catalytic activity of the ultrathin $\mathrm{CeO}_{2}$ sheets with numerous surface pits exhibited (Figure 6b) CO conversion of $50 \%$ at
$131{ }^{\circ} \mathrm{C}$, which was significantly superior than the pristine ultrathin $\mathrm{CeO}_{2}$ sheets $(3.2 \%)$ and bulk $\mathrm{CeO}_{2}(0.86 \%)$. Therefore, it can be concluded that, in addition to downsizing to ultrathin nanosheets, the introduced pits further promote the activity of the catalytic sites. As can be seen from Figure 6c, the lower activation free energy of ultrathin $\mathrm{CeO}_{2}$ nanosheets with numerous pits, as compared to ultrathin $\mathrm{CeO}_{2}$ nanosheets without pits confinement and bulk $\mathrm{CeO}_{2}$, brought the ability of $\mathrm{CO}$ oxidation at much lower temperature.

In the same vein, catalytic $\mathrm{CO}$ oxidation was reported ${ }^{[63]}$ on five atomic-layer-thick $\mathrm{SnO}_{2}$ sheets $(0.6 \mathrm{~nm}$ in thickness) obtained through hydrothermal reaction of $\mathrm{SnCl}_{2} \cdot 2 \mathrm{H}_{2} \mathrm{O}$ and ethylenediamine. The resulted high fraction of surface $\mathrm{Sn}$ and $\mathrm{O}$ atoms with lower coordination numbers than the interior atoms accounted for their improved catalytic properties that bestowed remarkably lower ignition temperature corresponding to $10 \% \mathrm{CO}$ conversion and overall conversion temperature of 
a

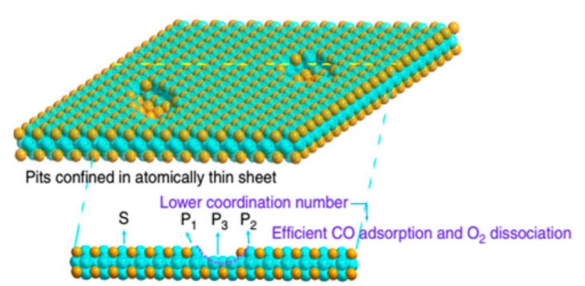

d

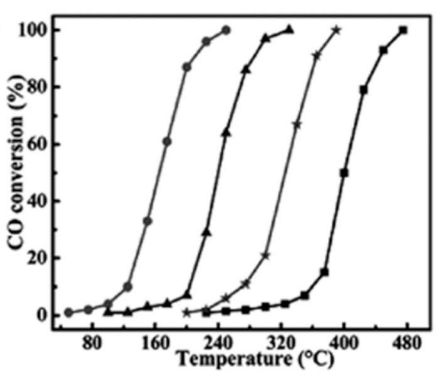

b

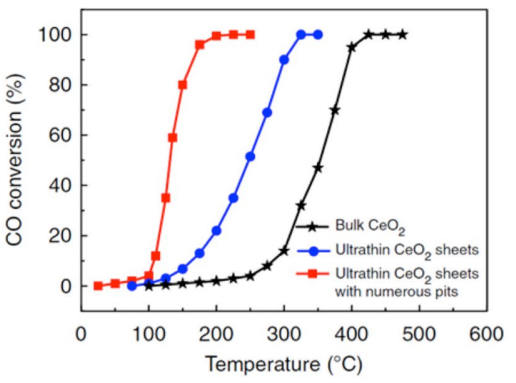

e

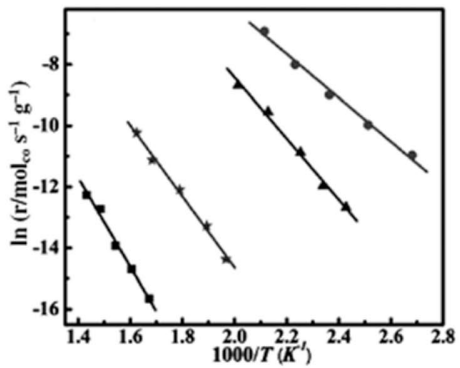

c

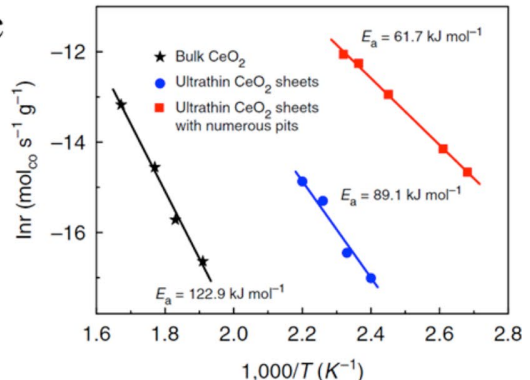

f

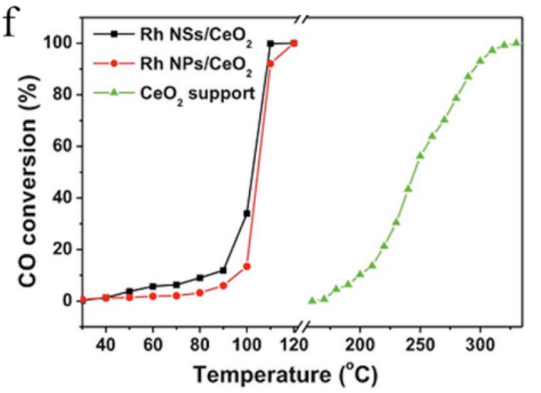

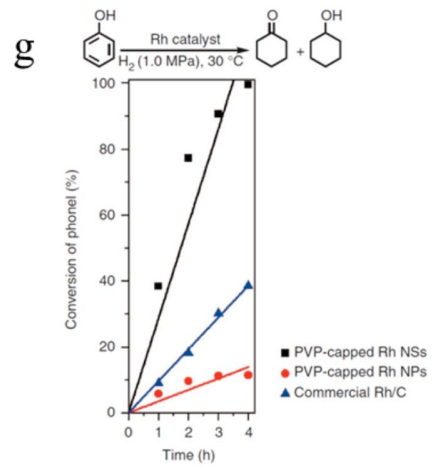

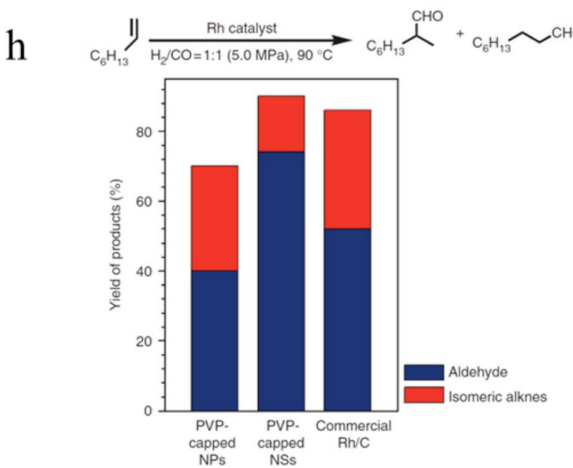

Figure 6. a) Atomically thin $\mathrm{CeO}_{2}$ sheets with surface-confined pits; $\mathrm{P} 1$ and $\mathrm{P} 2$ stand for the representative pit surrounding surface Ce sites, while $\mathrm{P} 3$ represents the bottom $\mathrm{Ce}$ site of the pit; $\mathrm{S}$ stands for the surface Ce site of atomically thin sheet and around the defects of bulk material. b) Catalytic activity for $\mathrm{CO}$ oxidation versus reaction temperatures (experimental error: $\pm 3 \%$ ) and c) the corresponding Arrhenius plot for the three $\mathrm{CeO}_{2}$ samples (experimental error: $\pm 3 \%$ ). (a-c) Reproduced with permission. ${ }^{[21]}$ Copyright 2013 , Nature Publishing Group. d) Catalytic activity for CO oxidation vs reaction temperatures and (e) corresponding Arrhenius plot of $0.66 \mathrm{~nm} \mathrm{SnO} 2$ nanosheets $(\bullet), 1.9 \mathrm{~nm} \mathrm{SnO} 2$ nanosheets $(\mathbf{\Delta})$, SnO $\mathrm{Sn}_{2}$ nanoparticles $(\star)$, and bulk $\mathrm{SnO}_{2}(\boldsymbol{\square})$. (d and e) Reproduced with permission. ${ }^{[63]}$ Copyright 2013, Wiley. f) CO conversion over Rh nanocatalysts loaded on $\mathrm{CeO}_{2}$ (black spot: Rh NSs synthesized by formaldehyde as reductant; red spot: Rh particles synthesized by acetaldehyde as reductant; green spot: the $\mathrm{CeO}_{2}$ support). Reproduced with permission. ${ }^{[44]}$ Copyright 2013, Royal Soceity of Chemistry. g) Hydrogenation of phenol and h) hydroformylation of 1-octene over ultrathin Rh nanosheet. Reproduced with permission. ${ }^{[13]}$ Copyright 2014, Nature Publishing Group.

$124{ }^{\circ} \mathrm{C}$ and $250{ }^{\circ} \mathrm{C}$, respectively, as compared to 1.9-nm-thick $\mathrm{SnO}_{2}$ sheet $\left(203{ }^{\circ} \mathrm{C}\right.$ and $\left.330{ }^{\circ} \mathrm{C}\right), \mathrm{SnO}_{2}$ nanoparticales $\left(270{ }^{\circ} \mathrm{C}\right.$ and $390{ }^{\circ} \mathrm{C}$ ) and bulk $\mathrm{SnO}_{2}\left(360{ }^{\circ} \mathrm{C}\right.$ and $\left.475{ }^{\circ} \mathrm{C}\right)$.(Figure $\left.6 \mathrm{~d}\right)$ Moreover, the existing large fraction of coordination-unsaturated surface atoms can be corroborated from the recorded smallest value of the apparent activation free energy which furthure reveals fast reaction occurring on the exposed surface of $0.66 \mathrm{~nm}$ thick $\mathrm{SnO}_{2}$ sheets (Figure 6e). Apart from metal oxides, 2D ultrathin metal nanosheets with unsaturated atoms on the surface were also introduced for catalysis of CO conversion. ${ }^{[11,13,44,45]}$ The report by Hou and co-workers is a typical example of this feature where they demonstrated ultrathin $1 \mathrm{~nm}$ thick $\mathrm{Rh}$ nanosheets supported on $\mathrm{CeO}_{2}$ to exhibit enhanced performance in the catalytic oxidation of $\mathrm{CO}$ as compared to $\mathrm{Rh}$ nanoparticles as depicted in Figure 6f. ${ }^{[4]}$ Meanwhile, Li's group reported the solvothermal synthesis of ultrathin rhodium nanosheets being highly active for catalytic hydrogenation and hydroformylation reactions. ${ }^{[13]}$ They studied hydrogenation of phenol and hydroformylation of 1-octene over three catalysts namely PVP capped Rh nanosheets, Rh nanopaticles and commercial $\mathrm{Rh} / \mathrm{C}$. The ultrathin nanosheets, due to the maximal exposure of $\mathrm{Rh}$ atoms with unsaturated coordination, resulted in $99.9 \%$ conversion within $4 \mathrm{~h}$ at near room temperature $\left(30^{\circ} \mathrm{C}\right)$ under $1.0 \mathrm{MPa} \mathrm{H}_{2}$ pressure for hydrogenation of phenol. Notably, the nanosheets also exhibited superior performance in catalyzing hydroformylation of 1-octene under mild reaction conditions (Figure $6 \mathrm{~g}$ and $\mathrm{h}$ ). The active role of metal nanosheets in the catalysis of organic reactions can be further pronounced from freestanding hexagonal palladium nanosheets which exhibited improved electrocatalytic oxidation of formic 

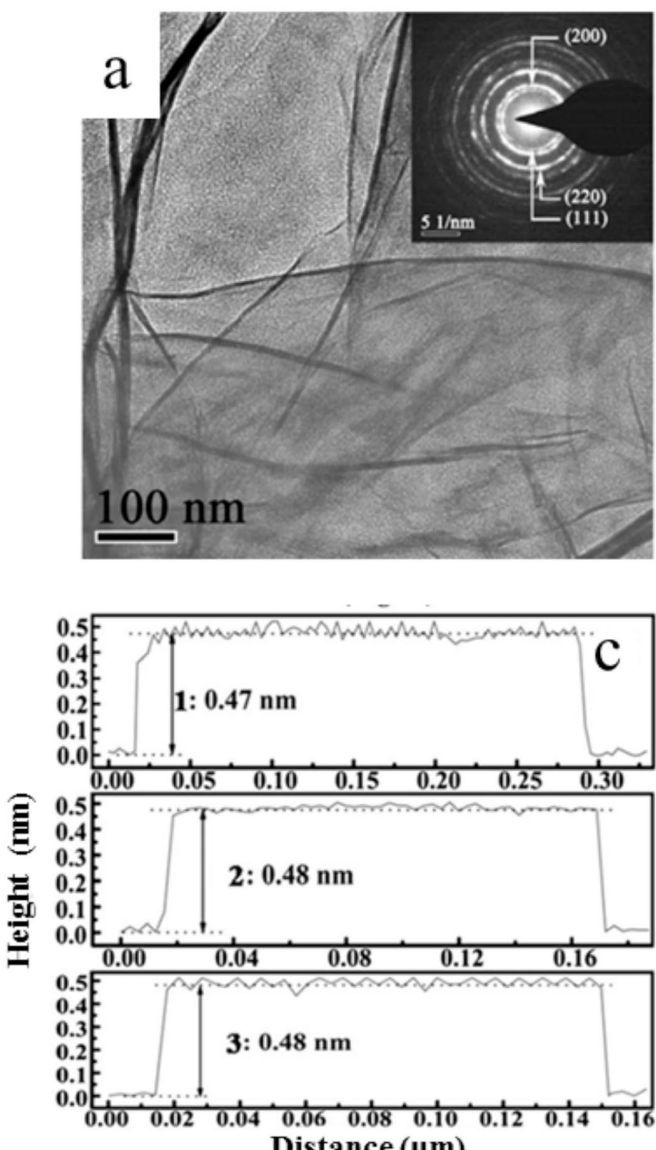

Distance $(\mu \mathrm{m})$

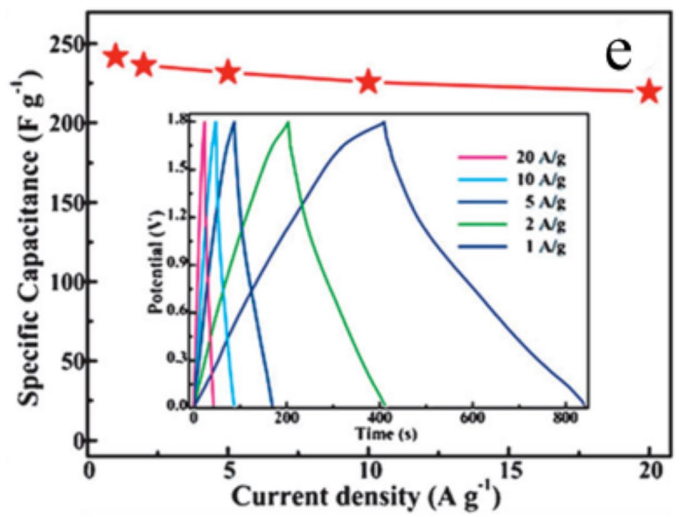

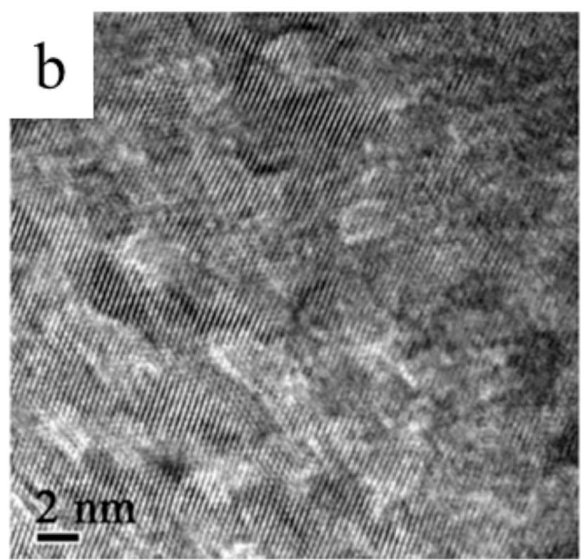
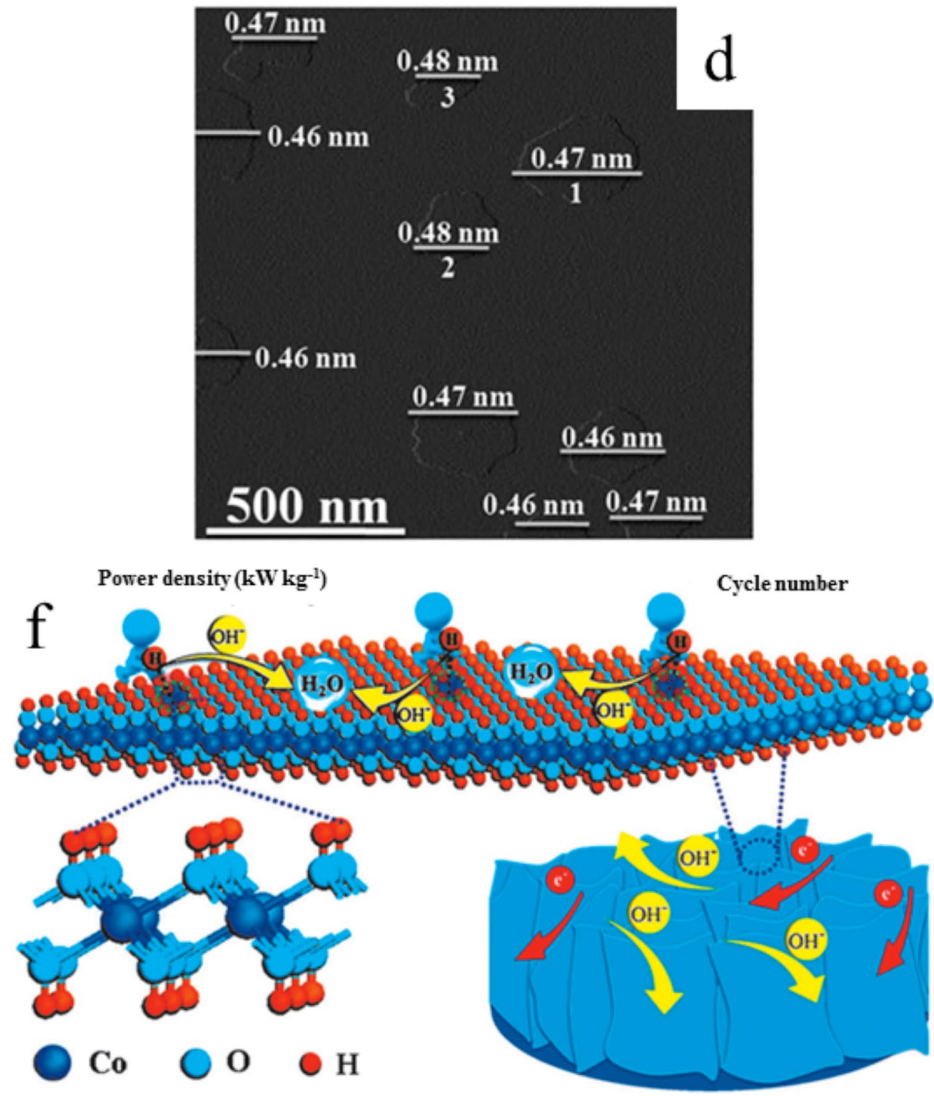

Figure 7. a) TEM image (the inset showing SAED pattern) and b) a planar HRTEM image of ultrathin NiO nanosheets. (a and b) Reproduced with permission. ${ }^{[50]}$ Copyright 2014, Nature Publishing Group. c) Height profiles derived from the atomic force microscopy (AFM) image in (d). The numbers 1 to 3 in (c) correspond to 1 to 3 in (d). e) Galvanostatic charge-discharge curves at different current densities (inset) and the corresponding calculated specific capacitances of the single-layer b-Co(OH) $)_{2}$ all-solid-state asymmetric supercapacitor. f) Schematic illustration of the hydrogen adsorption/ desorption process in the single-layer $\beta-\mathrm{Co}(\mathrm{OH})_{2}$ electrode. (c-f) Reproduced with permission. ${ }^{[65]}$ Copyright 2016, Wiley.

acid even outperforming the commercial palladium black catalyst by 2.5 times. ${ }^{[11]}$

The reactions of catalysis are only limited on the surfaces or near surfaces, and the bulks are inactive, which makes the low dimensional nanostructures possessing large specific surface area ideal candidates. Combining the short electron diffusion path and the improved structural stability, the 2D structure may be the most promising morphology for catalysis. More importantly, unlike 2D layered materials, the 2D non-layered materials of the same dimension are most frequently characterized by dangling bonds on their surface. This merit of numerous active sites accounts for further advantageous structural feature in catalysis. This endows them an ideal aspect to be considered as a promising catalysts for water splitting, CO conversion and other organic reactions. 


\subsection{Energy Storage and Conversion}

With the properties of high power density and rapid charge/discharge rate, supercapacitors are considered to be the potential energy storage devices in the future. ${ }^{[64]}$ Presently, some ultrathin 2D non-layered metal hydroxides ${ }^{[50,65]}$ and oxides ${ }^{[50]}$ have been utilized as the electrodes of supercapacitors. In this regard, atomically thin $\beta-\mathrm{Co}(\mathrm{OH})_{2}$ (Figure $\left.7 \mathrm{c}-\mathrm{d}\right)^{[65]}$ and ultrathin $\mathrm{NiO}$ nanosheets with the thickness of $1.16 \mathrm{~nm}$ (Figure $7 \mathrm{a}-\mathrm{b})^{[50]}$ were prepared via $2 \mathrm{D}$ oriented attachment and template-directed synthesis method. These atomically thick 2D nanostructures will provide a short ion and electron diffusion path distance during the charge and discharge process. In addition, it can enhance the capacity of the electrodes due to the large amount of electrochemical active sites and electrode-electrolyte interfaces. Hence, compared with $7 \mathrm{~nm}$ thick $\beta$ - $\mathrm{Co}(\mathrm{OH})_{2}$ nanosheets and bulk plates, the single-layered $\beta$ - $\mathrm{Co}(\mathrm{OH})_{2}$ nanosheets exhibited the highest specific capacitance of $2028 \mathrm{~F} \mathrm{~g}^{-1}{ }^{[65]}$ Based on the as-synthesized $\beta$ - $\mathrm{Co}(\mathrm{OH})_{2}$ nanosheets and $\mathrm{N}$-doped graphene, an all-solid-state asymmetric supercapacitor (ASC) was fabricated. Its typical charge-discharge curves and specific capacitances at different current densities shown in Figure 7e displayed a nearly linear variation with potential, demonstrating the capacitor-like behavior. This solid device exhibited specific capacitances of 241.9, 236.3, 231.8, 225.7 and $219.6 \mathrm{~F} \mathrm{~g}^{-1}$ at the current density of $1,2,5,10$ and $20 \mathrm{~A} \mathrm{~g}^{-1}$, respectively. The considerable capacity of single-layered $\beta$ - $\mathrm{Co}(\mathrm{OH})_{2}$ nanosheets can be assigned to its ultrathin thickness and the 100\% exposure to hydrogen atoms (Figure $7 \mathrm{f}$ ), which greatly favors the electrochemical redox reaction. The ultrathin $\mathrm{NiO}$ nanosheets based electrode also delivered a high specific capacitance of $2236 \mathrm{~F} \mathrm{~g}^{-1}$ at $0.5 \mathrm{~A} \mathrm{~g}^{-1}$ and remarkable stability with $99.1 \%$ retention after 2000 cycles. ${ }^{[50]}$ The desirable architecture, maximized active material utilization and favorable reaction kinetics endow this electrode prominent electrochemical performance.

Besides supercapacitors, the rechargeable Li-ion batteries (LIBs) based on 2D non-layered materials have also been extensively studied. ${ }^{[51,55,66]}$ In classical Li-ion batteries, the energy charge and discharge rely on the intercalation reactions for which $\mathrm{Li}^{+}$ions are inserted into (or extracted from) an open host structure (interlamination of the electrodes) with a concomitant addition (or removal) of electrons. However these LIBs suffer from the disability of further improving the capacity because of these electrode materials cannot incorporate more than one Li-ion per metal oxides. To overcome this bottleneck, other electrode materials that take advantage of conversion reaction mechanism have been proposed recently. ${ }^{[67]}$ The comparison of the different mechanisms using $\mathrm{Co}_{3} \mathrm{O}_{4}$ as an example is shown in Figure 8b. As can be seen, this reaction mechanism is wholly Li-ion surface storage and the performance of these new materials is strongly dependent on the surface area and quality, which makes the bulk materials unsuitable. Keeping this in mind, it is quite reasonable to think of downsizing the dimensions of the electrode materials. In particular, with short ion and electron diffusion path distances, large electrochemical active sites and electrode-electrolyte interface, and improved structural stability, 2D metal oxide nanomaterials with single or few layer thickness have been recognized as novel electrode materials. ${ }^{[51,66]}$ As such, the Li-ion charge and discharge in these species is on the surfaces, which meets the fast Li-ion storage in the batteries. Meanwhile, the high interfacial contact area with high Li-ion flux cross the interface contributes to the fast interfacial charge transfer and minimize the time constant of Li-ion diffusion. ${ }^{[68]}$ These advantages of the usage of 2D non-layered materials in Li-ion batteries are demonstrated in atomically thick $\mathrm{Co}_{3} \mathrm{O}_{4}$ nanosheets as shown in Figure 8a. ${ }^{[51]}$ These results suggest the configuration of atomically thick nanosheets is comprehensively superior in optimizing lithium storage ability. The galvanostatic discharge-charge profiles of $\mathrm{Co}_{3} \mathrm{O}_{4}$ nanosheets and nanoparticles shown in Figure 8c and $8 \mathrm{~d}$ reveal the ultrahigh initial discharge capacity $\left(>1350 \mathrm{~mA} \mathrm{~h} \mathrm{~g}^{-1}\right)$ and low irreversible capacity loss (19\%). Notably, a very high and stable reversible capacity of $1291.2 \mathrm{~mA} \mathrm{~h} \mathrm{~g}^{-1}$ with $0.23 \%$ loss per cycle is also achieved (Figure 8d). For comparison, the electrode based on the $\mathrm{Co}_{3} \mathrm{O}_{4}$ nanoparticles exhibited rapid capacity fading. The rate capacities of different electrodes at various charge-discharge rates are shown in Figure 8e and $8 \mathrm{f}$. The ultrathin $\mathrm{Co}_{3} \mathrm{O}_{4}$ nanosheets for fast lithium storage is quite superior than that of nanoparticles. During the measurement, the obtained capacity at each cycling rate remains relatively constant and exhibits $1308.6 \mathrm{~mA} \mathrm{~h} \mathrm{~g}^{-1}$ at $0.1 \mathrm{C}, 1011.1 \mathrm{~mA} \mathrm{~h} \mathrm{~g}^{-1}$ at $0.2 \mathrm{C}, 1022.0 \mathrm{~mA} \mathrm{~h} \mathrm{~g}^{-1}$ at $0.5 \mathrm{C}$ and $937.8 \mathrm{~mA} \mathrm{~h} \mathrm{~g}^{-1}$ at $1 \mathrm{C}$, respectively (Figure $8 \mathrm{e})$. At the same time, J. Shen et al ${ }^{[66]}$ and C. Wang et al. ${ }^{[55]}$ also prepared single-crystal $\alpha-\mathrm{Fe}_{2} \mathrm{O}_{3}$ nanodiscs and $\mathrm{SnO}_{2}$ nanosheets for improving the lithium storage capacity.

\subsection{Optoelectronic Devices}

Optoelectronic devices, including photodetectors, solar cells and LEDs, etc., are electric devices that can detect, generate, and interact with or control light. The property and performance of an optoelectronic device are strongly dependent on the structures of the functional materials. For example, compared with bulk materials, nanomaterials usually harvest light more efficiently due to their high specific surface area. In addition, the specific structure of nanomaterials also strongly affects the electronic spectrum of charge carriers then the optoelectronic properties. For instance, the density of states changes from discrete levels for zero-dimensional quantum dots to a saw-like quasicontinuum in one-dimentional nanorods, and finally to step-like quasicontinum in two-dimension nanoplates. ${ }^{[1]}$ As a consequence, optoelectronic devices have been one of the most promising applications of nanostructured materials ever since its emergence. This is especially true for nanomaterials with two-dimensional configurations because of their high compatibility with traditional device fabrication process as well as flexible substrates. The recently emerged 2D layered materials, like $\mathrm{MoS}_{2}$, provide a good example. ${ }^{[7,69]}$ Unfortunately, only limited numbers of materials possess layered structure and most of the materials with outstanding optical properties are covalently bonded in three-dimension. Thanks to the synthesis methods discussed above, a growing number of 2D non-layered materials, including metal chalcogenides, like $\mathrm{PbS}^{[22]}$ and $\mathrm{CdS} / \mathrm{Se}^{[1]}$ metal oxides, like $\mathrm{TiO}_{2}$ and $\mathrm{ZnO},{ }^{[20]}$ topological crystalline insulators, like $\mathrm{Pb}_{x} \mathrm{Sn}_{1-x} \mathrm{Se},{ }^{[32]}$ and organic-inorganic hybrid 
a

high interfacial contact with electrolyte

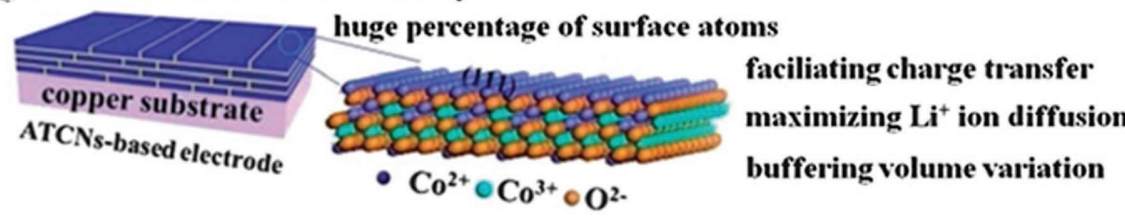

b

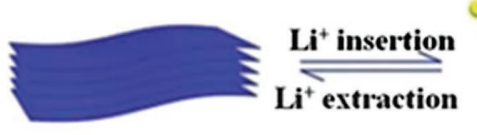

atomically-thick nanosheets

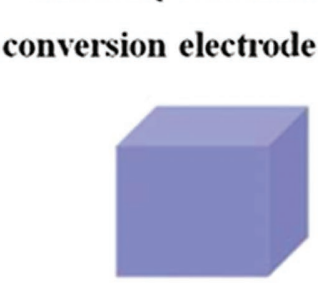

bulk counterpart
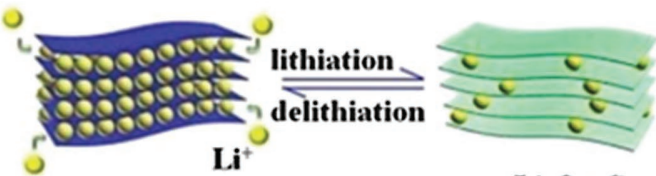

$\mathrm{Li}_{2} \mathrm{O}+\mathrm{Co}$

$8 e^{\text {discharge }} \frac{}{\text { Charge }} 3 \mathrm{Co}+4 \mathrm{Li}_{2} \mathrm{O}$

C
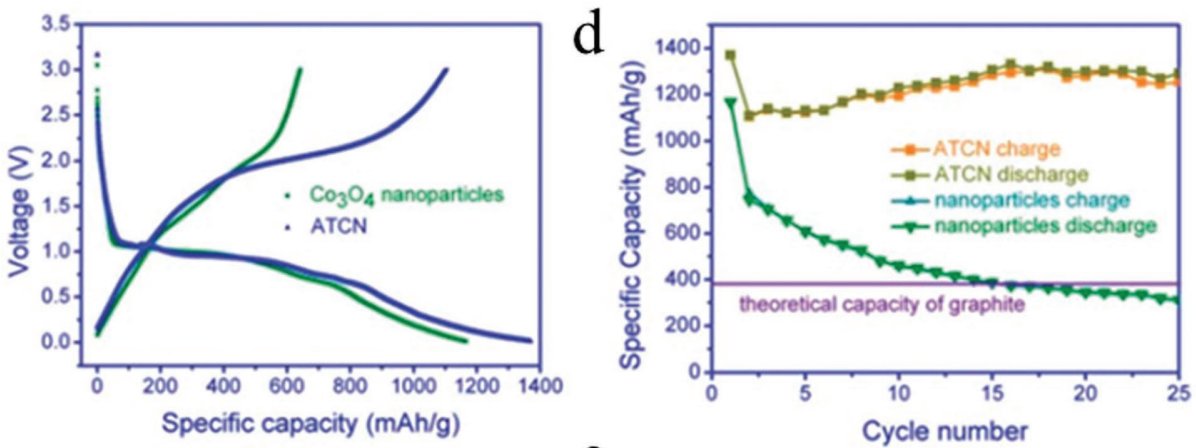

e

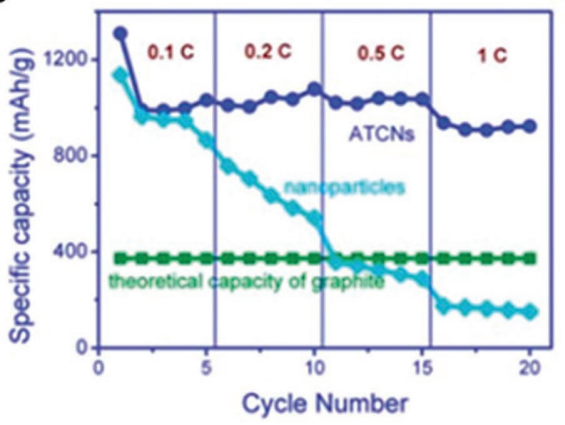

f
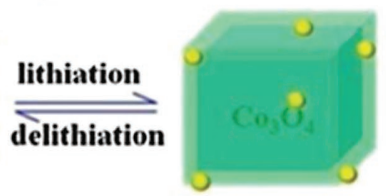

$\mathrm{Li}_{2} \mathrm{O}+\mathrm{Co}$

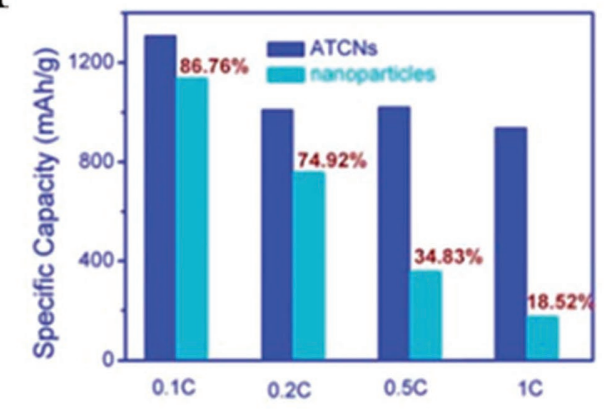

Figure 8. a) Advantages of using atomically thick $\mathrm{Co}_{3} \mathrm{O}_{4}$ nanosheets (ATCNs) on a copper substrate as a conversion electrode for rechargeable LIBs. b) Schematic illustration of the working mechanism of conversion electrode materials using atomically thick nanosheets and their bulk counterpart. The synergistic advantages endow atomically thick nanosheets eminently enhanced lithium storage performance. c) The initial galvanostatic discharge-charge profiles of ATCNs and nanoparticles at a rate of $0.5 \mathrm{C}\left(1 \mathrm{C}=890 \mathrm{~mA} \mathrm{~g}^{-1}\right)$. d) The cycling life of ATCNs and nanoparticles at a rate of 0.5 C. (e) and (f) Cycling performance of ATCNs at different charge-discharge rates compared to nanoparticles. (a-f) Reproduced with permission. ${ }^{[51]}$ Copyright 2014, RSC.

perovskites, like $\mathrm{CH}_{3} \mathrm{PbI}_{3}$, ${ }^{[34,35]}$ have been developed for optoelectronic devices. In this part, we will give a brief review about optoelectronics based on 2D non-layered materials, emphasizing on photodetectors and solar cells.
The initial trial was using $\mathrm{PbS}$ nanosheets for photodetection. In 2010, H. Weller's group developed a new self-assembly strategy of synthesizing ultrathin $\mathrm{PbS}$ nanosheets with the lateral dimensions on the scale of micrometers (see the synthesis 

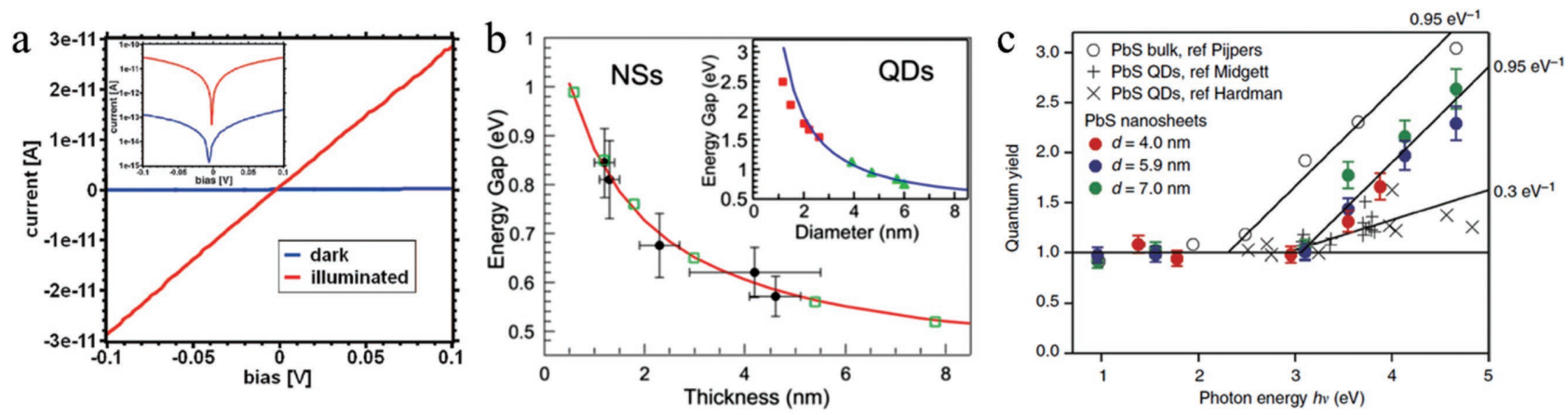

d
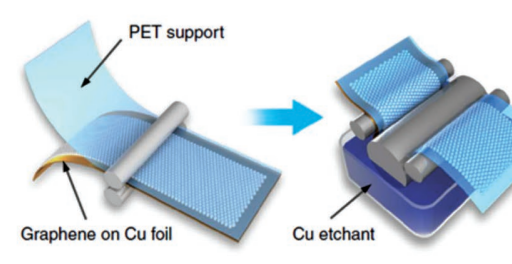

Graphene on

PET support

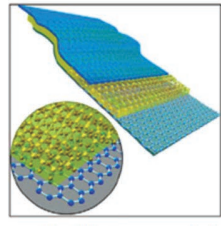

Flexible, transparent photodetector electrode

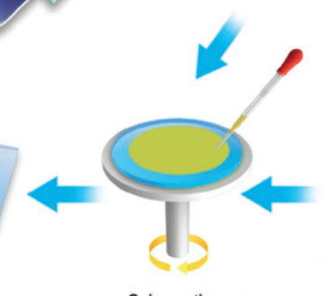

Spin coating
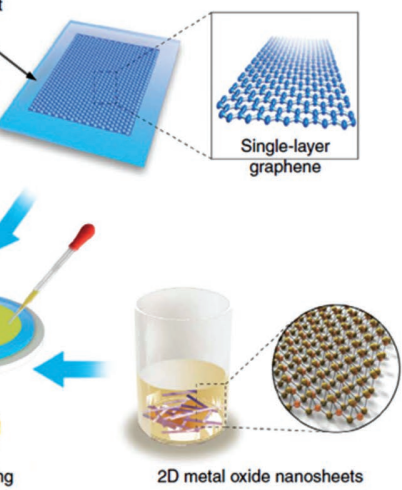

e

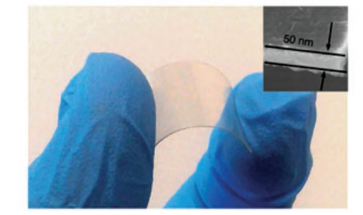

f

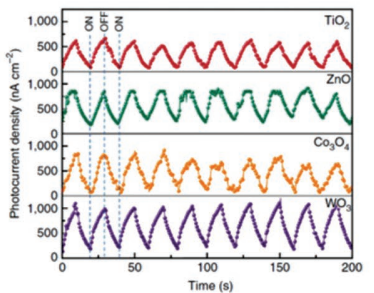

Figure 9. a) Current-voltage curves of PdS nanosheets with (red line) and without (blue line) illumination with $532 \mathrm{~nm}$ laser. The inset shows the curves in logarithmic scale. Reproduced with permission. ${ }^{[22]}$ Copyright 2010, AAAS. b) Dependence of band gaps (solid dots: experimental results measured from PL spectra; green squares: data got from theoretical calculation) of PbS nanosheets on their thickness. The inset gives the corresponding relationship of PbS quantum dots. Reproduced with permission. ${ }^{[25]}$ Copyright 2014, American Chemical Society. c) Quantum yield plotted versus pump photon energy for PbS nanosheets with thickness as indicated. Reproduced with permission. ${ }^{\left[{ }^{[1]}\right]}$ Copyright 2014, Nature Publishing Group. d) Schematic diagram of the fabrication of the photodetector based on as-synthesized 2D metal oxides. e) Optical image of the as-fabricated photodetector with inset showing the SEM image of its cross section. f) The photoreponse behavior of the photodetector composed with different kinds of 2D metal oxides with altering on and off ultraviolet light. (d-f) Reproduced with permission. ${ }^{[20]}$ Copyright 2014, Nature Publishing Group.

method part for detail), which are large enough so can be integrated in a photodetector demo device. ${ }^{[22]}$ With the help of chlorine-containing solvents, the assembled $\mathrm{PbS}$ nanosheet are lack of ligands in the in-plane dimensions and show outstanding photoresponse properties with responsivity of $0.472 \mathrm{~A} / \mathrm{W}$ under the illumination of $532 \mathrm{~nm}$ laser, as shown in Figure 9a. While scaling the dimension(s) of semiconductors down to nanoscale (usually $<10 \mathrm{~nm}$ ), quantum confinement will show out. One of the most obvious phenomena is the increasing energy band gap $\left(E_{\mathrm{g}}\right)$ with decreasing dimensions. 2D non-layered materials are no exception. In 2011, S. Ithurria et al. synthesized CdSe, CdS and CdTe nanoplates with thickness of 4-11 monolayers. ${ }^{[1]}$ Due to their atomically flat surfaces, strong dependences of $E_{\mathrm{g}}$ on thickness were observed in the nanoplates, which are well described by the PidgeonBrown effective mass approximation model. Similar trend but with different dependence was observed in $\mathrm{PbS}$ nanosheets. As shown in Figure 9b, using the same synthesis method developed by $\mathrm{H}$. Weller's group, Ghadendra B. Bhandari et al. found a $E_{\mathrm{g}} \sim 1 / L$ relationship in $2 \mathrm{D} \mathrm{PbS}$ which was attributed to the breakdown of effective mass approximation. ${ }^{[25]}$ It is therefore possible to design photodetectors with better light harvesting ability through choosing nanosheets with suitable thickness. Besides $E_{\mathrm{g}}$, carrier multiplication is another important process that determines the photo-electronic conversion efficiency of photodetectors. ${ }^{[70]}$ In semiconductors, hot electron-hole pairs will generate initially while illuminated by a light with photon energy lager than $E_{\mathrm{g}}$. The excess energy then can be relaxed through stimulating new electrons across the band gap and generating new electron-hole pairs. The process goes on until the hot electron-hole pairs fully cool down to energetically equivalent state. As a result, one photon will generate more than one electron-hole pair (which is also known as carrier multiplication). Due to the strong Coulomb interaction, relative low carrier multiplication efficiency is usually observed in low dimensional structures, like quantum dots. However, this is not the case for 2D geometry. Using a similar method with H. Weller group, Michiel Aerts et al. synthesized $\mathrm{PbS}$ nanosheets and measured the dynamics of photogenerated charge carriers by time-resolved laser pump-probe optical absorption facilities. ${ }^{[71]}$ Notably as shown in Figure 9c, the $\mathrm{PbS}$ nanosheets show comparable quantum efficiency with bulk $\mathrm{PbS}$, meaning that, unlike the quantum dots, nanorods and bulk state, the excess energy of hot electron-hole pairs is completely used to generate additional charge carries in the 2D scenario. 

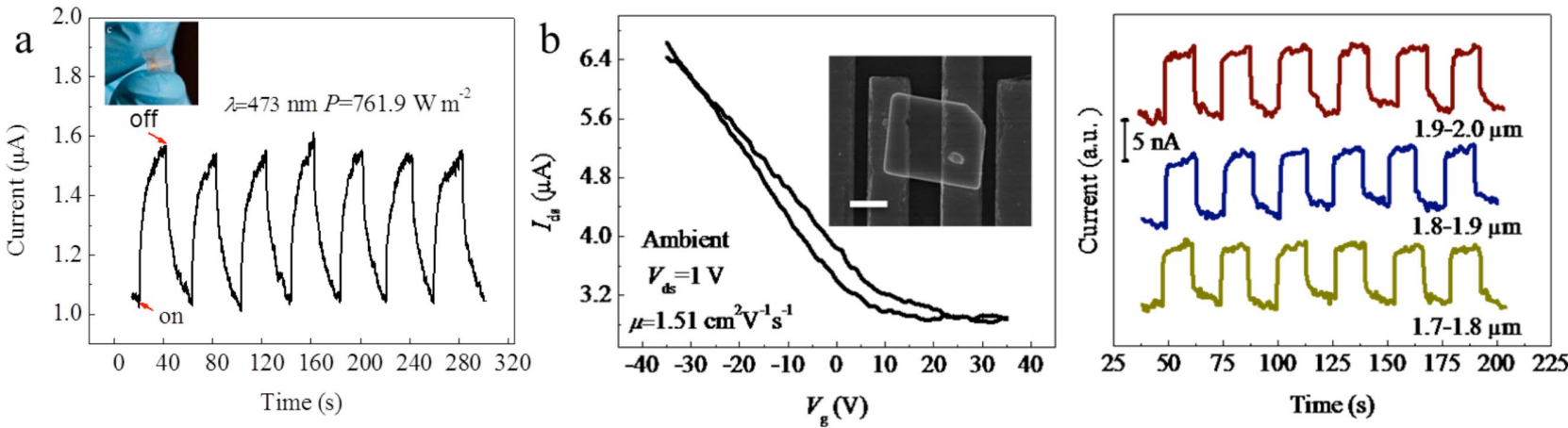

c
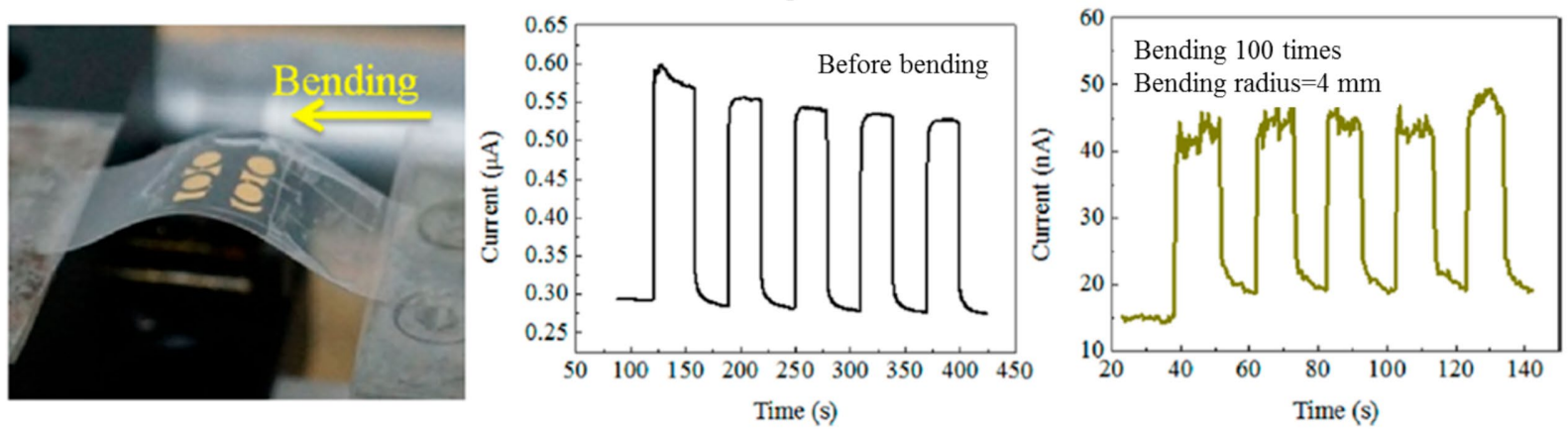

d
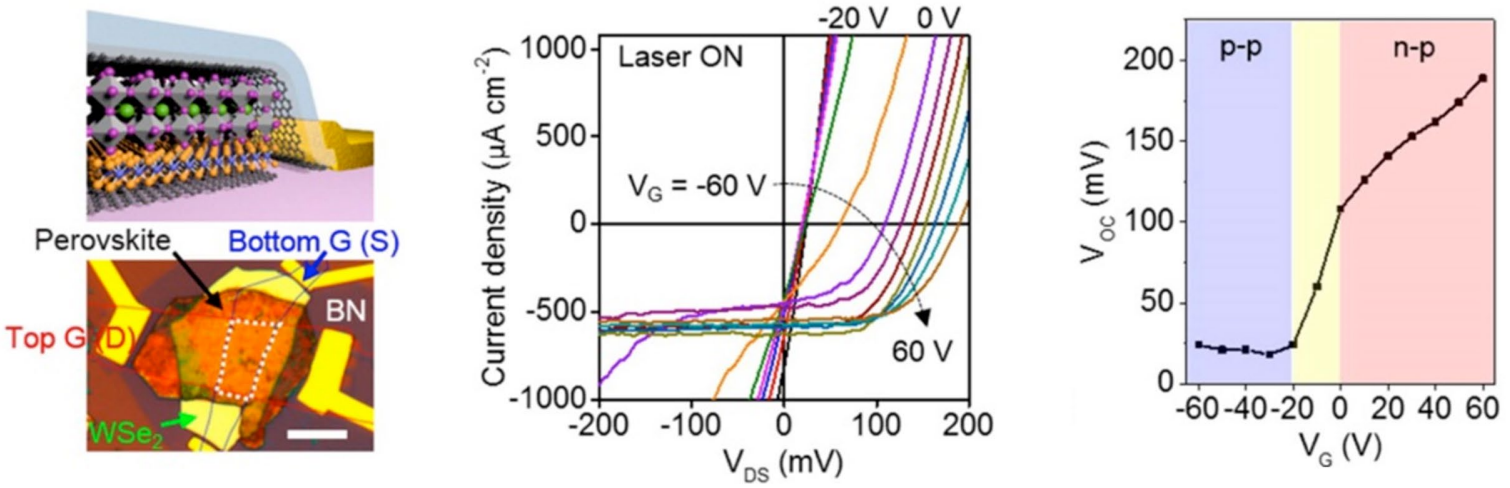

Figure 10. a) Time-dependent photoresponse of the Te nanoplates. The inset shows the photograph of the device. Reproduced with permission. ${ }^{[37]}$ Copyright 2014, American Chemical Society. b) Left panel, transfer characteristic of $\mathrm{Pb}_{1-x} \mathrm{Sn}_{x} \mathrm{Se}$ nanoplate with inset showing the SEM image of the device. Right panel:mid-infrared detection of the $\mathrm{Pb}_{1-x} \mathrm{Sn}_{x}$ Se nanoplates at 1.7-2.0 $\mu \mathrm{m}$. Reproduced with permission. ${ }^{[33]}$ Copyright 2015, Wiley. $\mathrm{c}$ ) Left panel, Photograph of the $2 \mathrm{D} \mathrm{Pb}{ }_{1-x} \mathrm{Sn}_{x} \mathrm{Se}$ nanoplates-based device while bending. Time-dependent photoresponse behavior of the device before (middle panel) and after (right panel) 100 times of bending. Reproduced with permission. ${ }^{[32]}$ Copyright 2015, American Chemical Society. d) Left panel, schematic illumination and optical microscopy image of the graphene/WSe 2 /perovskite/graphene device. Middle panel, the drain current-bias voltage curves obtained under $532 \mathrm{~nm}$ laser illumination with varied gate voltages. Right panel, the gate-dependent open-circuit voltage extracted from the middle panel. Reproduced with permission. ${ }^{[35]}$ Copyright 2016, American Chemical Society.

Photodetectors were also achieved on 2D metal oxides. Recently, Ziqi Sun et al. developed a generalized surfactantassistant self-assembly synthesis method for ultrathin 2D transition metal oxides, through which $\mathrm{TiO}_{2}, \mathrm{ZnO}, \mathrm{Co}_{3} \mathrm{O}_{4}, \mathrm{WO}_{3}$, $\mathrm{Fe}_{3} \mathrm{O}_{4}$ and $\mathrm{MnO}_{2}$ nanosheets with thickness of about $200 \mathrm{~nm}$ and lateral dimensions ranging 1 to $10 \mu \mathrm{m}$ were synthesized successfully. ${ }^{[20]}$ Using the as-synthesized 2D transition metal oxides nanosheets, graphene and polyethylene terephthalate (PET) as working materials, electrodes and substrates, flexible ultraviolet (UV) photodetectors were fabricated. Figure 9d and e show the schematic diagram of their fabrication process and the optical image of the final device, respectively. The as fabricated photodetectors based the variable 2D metal oxides all show good response to visible-light-blind ultraviolet (UV-A band at $320-400 \mathrm{~nm}$ ) light as shown in Figure 9f.

All the above photodetectors are based on 2D non-layered materials synthesized by wet chemistry methods, which suffer from small lateral dimensions and relative poor crystalline quality. Hence, most of the researches are restricted to spectroscopic study or devices constructed with a pile of randomly distributed 2D non-layered material flakes, in which the novelty of individual 2D non-layered single crystal has been ignored. Taking advantage of vdWE method, our group recently synthesized 2D metal chalcogenides, like Te and PbSnSe, and studied the corresponding photoresponse properties of individual nanoflakes. ${ }^{[32,37]}$ Single crystalline Te nanoplates with 
thickness of $30-80 \mathrm{~nm}$ and lateral sizes of $6-10 \mu \mathrm{m}$ were firstly synthesized on mica substrates. ${ }^{[37]}$ Then photodetectors were fabricated directly on the mica sheets by shadow mask method. As shown in Figure 10a, they show good response to $473 \mathrm{~nm}$ laser with responsivity up to $389.5 \mathrm{~A} \mathrm{~W}^{-1}$. To be noted, the dangling bond free mica sheets not only act as a substrate for vdWE, more importantly, their good flexibility also provides a platform to in situ fabricate pliable photodetectors. For example, as shown in Figure $10 \mathrm{c}, \mathrm{Pb}_{1-x} \mathrm{Sn}_{x} \mathrm{Se}$ photodetecors on mica sheet only show a small decrease of photocurrent, which is attributed to the deteriorate metal contacts, and still exhibit good stability even after 100 times of bending. ${ }^{[35]}$ Replacing the role of mica sheets by $2 \mathrm{D}$ layered materials, $2 \mathrm{D} \mathrm{Pb}_{1-x} \mathrm{Sn}_{x} \mathrm{Se}$ nanoplates were grown on $\mathrm{SiO}_{2} / \mathrm{BN}$ substrates. ${ }^{[33]} \mathrm{Pb}_{1-x} \mathrm{Sn}_{x} \mathrm{Se}$ is a direct narrow band gap semiconductor with great application potential in infrared detection and thermoelectric conversion. The bulk material exhibits highly efficient photodetection in the range of 3-5 $\mu \mathrm{m}$ wavelength which matches with mid-infrared atmosphere window that is an important communication waveband. For the 2D $\mathrm{Pb}_{1-x} \mathrm{Sn}_{x} \mathrm{Se}$ nanoplates, as shown in Figure 10b, room-temperature field effect mobility of $0.73-4.90 \mathrm{~cm}^{2} \mathrm{~V}^{-1} \mathrm{~s}^{-1}$ that is comparable with some layered materials and molecular crystals and more importantly, the $\mathrm{Pb}_{1-x} \mathrm{Sn}_{x} \mathrm{Se}$ nanoplates show high-efficient room-temperature photoresponse with detection wavelength extending to 1.9-2.0 $\mu \mathrm{m}$ which matches with one of the infrared atmospheric windows $(1.5-1.9 \mu \mathrm{m})$, suggesting its great applications potential in military communication, environmental monitoring, and remote sensing (Figure 10b).

Another emerging application of 2D non-layered materials is solar cells based on 2D organic-inorganic hybrid perovskites (organometal halide perovskites), i.e., $\mathrm{CH}_{3} \mathrm{NH}_{3} \mathrm{PbX}_{3}$, where $\mathrm{X}$ represents halides $\left(\mathrm{Cl}, \mathrm{Br}\right.$ and I). ${ }^{[34,36,41]}$ After the pioneering works, organometal halide perovskites have attracted a great deal of attentions as light absorbers for thin film solar cells due to their strong absorption over visible to near-IR spectrum and ultra-long free carrier diffusion length (above $100 \mathrm{~nm}){ }^{[72]}$ Despite a short history, a record power conversion efficiency of above $20 \%$ has been achieved, demonstrating their very promising potential in practical application. ${ }^{[73]} \mathrm{Up}$ to now, two methods have been used to fabricate $\mathrm{CH}_{3} \mathrm{NH}_{3} \mathrm{PbX}_{3}$ films: spin-coating and thermal evaporation method, which unfortunately suffer from bad homogeneity and complexity of control, respectively. ${ }^{[34,74]}$ For photovoltaic effect, the final performances, such as open circuit voltage $\left(V_{o c}\right)$, filling factor $(F F)$ and power efficiency $(\eta)$, are strongly dependent on the generation, separation and transfer process of photo-generated charge carriers, which are fundamentally rely on the crystalline quality, including defects and domain sizes. ${ }^{[75]}$ For a higher performance, methods capable of synthesizing/growing organometal halide perovskites with better crystalline quality are needed. Recently, using template-assisted CVD method (see the methods discussion), Q. H. Xiong, ${ }^{[34]}$ Z. Liu, ${ }^{[36]}$ and X. F. Duang ${ }^{[35,41]}$ groups synthesized $\mathrm{CH}_{3} \mathrm{NH}_{3} \mathrm{PbX}_{3}$ nanoplates with thickness of tens to hundreds of nanometers and lateral dimensions up to tens of micrometers. The nanoplates were demonstrated to be high-quality single crystals with ultra-long carrier lifetimes. However, due to the solubility of organometal halide perovskites in many common solvents, like water, acetone and alcohol, the corresponding device fabrication and electronic property studies have been limited. In view of this problem and utilizing the recently developed dry-transfer method for van der Waals heterostructures, Hung-Chieh Cheng et al. fabricated $2 \mathrm{D}$ layered materials $/ \mathrm{CH}_{3} \mathrm{NH}_{3} \mathrm{PbI}_{3}$ hybrid structures and studied the corresponding optoelectronic properties. ${ }^{[35]}$ To be specific, $\mathrm{PbI}_{2}$ thin flakes were firstly exfoliated by the stand scotch method and then converted to $\mathrm{CH}_{3} \mathrm{NH}_{3} \mathrm{PbI}_{3}$ by methylammonium iodide vapor phase reaction. Graphene/perovskite/ graphene heterosrtcutures were fabricated and gate voltage tunable PV effects were observed which were attributed to the different Schottky barriers formed at the bottom graphene/perovskite and up graphene/perovskite interfaces. In addition, a p-type conduction behavior was observed for the $\mathrm{CH}_{3} \mathrm{NH}_{3} \mathrm{PbI}_{3}$ flakes, based on which the p-n/p-p heterostructure with $\mathrm{WSe}_{2}$ were constructed as shown in Figure 10d. Due to the ambipolar property of $\mathrm{WSe}_{2}$, the heterostructures exhibited a gate tunable PV effect with a maximum $V_{\text {oc }}$ of about $200 \mathrm{meV}$ (Figure 10d). At the time of writing, this is the only work about devices fabrication and characterization based on 2D organometal halide perovskites which unambiguously provide a possibility of studying the intrinsic property of thin organometal halide perovskite flakes and a way of combining 2D layered and non-layered materials together. Organic-inorganic hybrid perovskites are excellent, but their 2D structures are extremely less studied. However, considering the novelty that 2D geometry has shown, it is worthwhile to see what surprise $2 \mathrm{D}$, especially ultrathin, organometal halide perovskites would bring to us.

\subsection{Topological Crystalline Insulators}

Low-dissipation electronic devices and quantum communications based on topological crystalline insulators (TCIs) are another emerging application of 2D non-layered materials. One of the biggest limits of modern Si-based electronic devices is their high thermal dissipation that comes from the serious scattering of charge carriers from impurities and phonons during transport. Topological insulators (TIs), in which topological protection from time-reversal symmetry, strong spinorbit coupling (band inversion) and relativistic effects lead to the occurrence of gapless metallic state on the surfaces/edges, provide a promising alternative choice to solve this problem. ${ }^{[76]}$ Inspired by the novel properties of TIs, researchers have been trying to find new topological phases protected by other invariants. Topological crystalline insulators (TCIs), including SnTe, $\mathrm{PbTe}$ and $\mathrm{PbSe}$, as well as their ternary solid solutions, like $\mathrm{Pb}_{1-x} \mathrm{Sn}_{x} \mathrm{Te}$ and $\mathrm{Pb}_{1-x} \mathrm{Sn}_{x} \mathrm{Se}$, are recently discovered topological phase in which the role of time-reversal symmetry in TIs is replaced with point-group symmetry. ${ }^{[77,78]}$ The existence of TCIs was theoretically predicted by Liang Fu in 2011. ${ }^{[7]}$ They then manifested that SnTe exhibits this non-trivial topological state. ${ }^{[78]}$ As shown in Figure 11a, the band structure of SnTe are switched and inverted at $L$ points, providing the possibility of topological states. By studying the surface state, they found that there do exist Dirac cones at each of the high-symmetry crystal surfaces of SnTe, including (001), (110) and (111) planes, but with even numbers, which is profoundly different from the odd numbers of TIs. To illustrate the specificity of SnTe, the 
a
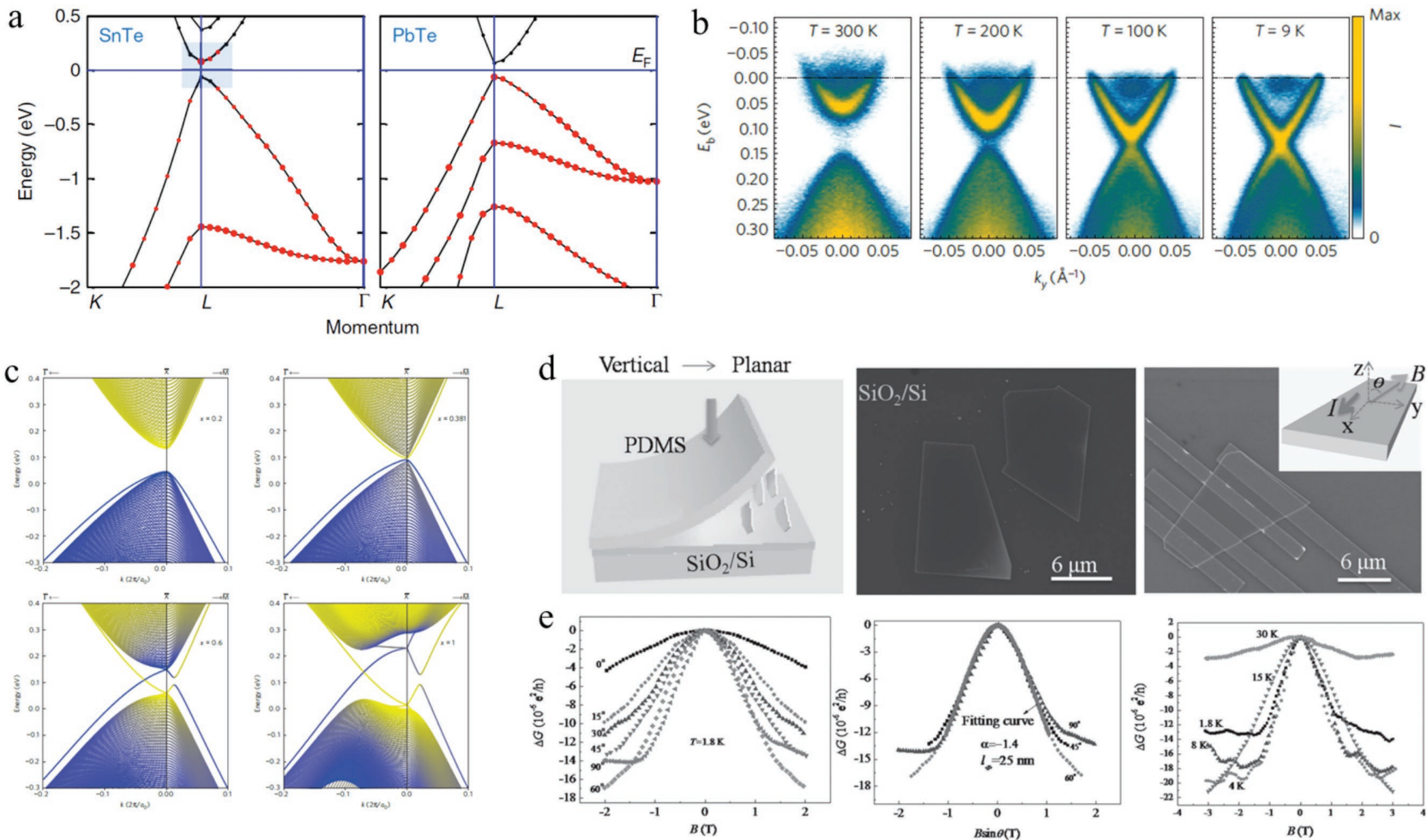

Figure 11. a) The electronic band structure of SnTe (left panel) and PbTe (right panel). Reproduced with permission. ${ }^{[78]}$ Copyright 2012, Nature Publishing Group. b) Temperature dependence of APES spectra of the $\mathrm{Pb}_{0.77} \mathrm{Sn}_{0.23}$ Se monocrystals. c) Theoretical tight-binding model calculations of the band structures of $\mathrm{Pb}_{1-x} \mathrm{Sn}_{x}$ Te thick slab with varying content of $x$. (b and c) Reproduced with permission. ${ }^{[79]}$ Copyright 2012, Nature Publishing Group. d) Left panel, schematic diagram showing the transfer pross of $\mathrm{Pb}_{1-x} \mathrm{Sn}_{x}$ Te nanoplates. SEM images of the as-transferred $\mathrm{Pb}_{1-x} \mathrm{Sn}_{x} \mathrm{Te}_{1} \mathrm{Panoplates}\left(\mathrm{middle}_{1}\right.$ panel) and four-terminal device (right panel). The inset shows the relation of magnetic field, current flow and $\mathrm{Pb}_{1-x} \mathrm{Sn}_{x} \mathrm{Te}$ nanoplates. e) Left panel, the change of magneto-conductance as the function of B at different angle at $2 \mathrm{~K}$. Middle panel, Magneto-conductance as the function of Bsin $\theta$ coincides with each other at low magnetic field $(-0.5$ to $0.5 \mathrm{~T})$. Right panel, temperature-dependent WAL effect which disappears at high temperature due to strong thermal scattering. (d and e) Reproduced with permission. ${ }^{\left[{ }^{[9]}\right.}$ Copyright 2016, Wiley.

band structure and surface states were also studied. As shown in the right panel of Figure 11a, PbTe shows no metallic Diraclike band, i.e., it's a naturally trivial insulator, due to the band dispersions. The prediction was then immediately confirmed by other three groups through angle-resolved photoemissionspectroscopy (APRES) in 2012. ${ }^{[79,80]}$ Compared with TIs, TCIs exhibit three intriguing features: (1) they are of highly symmetry crystal structures, (2) gapless metallic states only reside on those mirror-symmetry surfaces such as (100), (111), and (110), (3) Dirac cones on TCIs surface can be opened up by breaking symmetry, manipulating temperature, and tailoring compositions. ${ }^{[30]}$ For example, as shown in Figure $11 \mathrm{~b}$ and c, there will be a non-trivial to trivial topological phase transition while continuously varying the composition of $\mathrm{Pb}_{1-x} \mathrm{Sn}_{x} \mathrm{Te}$ or changing the temperature tested.

Controllable synthesis is always a premise but usually challenging step of studying any new physics from 'new' materials. This is especially true for TCIs because the fragile surface states are very easily overwhelmed by the strong bulk state. As a consequence, TCIs with low-dimensional structures becomes the mostly preferred morphology, in which the huge surfacevolume ratio ensures the successful detection of gapless surface states. Specially, the the large top and bottom surfaces of two-dimensional TCIs provide an excellent platform for topological surface states characterization. ${ }^{[30]} \mathrm{Up}$ to now, most of the initial studies are based on molecular beam epitaxy (MBE) method, by which the thickness and composition of the TCI films can be precisely controlled. However, it is expensive and time consuming. Stimulated by this condition, Cha et al. firstly grew SnTe nanoplates via chemical vapor deposition (CVD) method, which is much easier and cheaper compared with MBE. [31] Using $\mathrm{SnTe}$ powder, $\mathrm{SiO}_{2} / \mathrm{Si}$ and $\mathrm{Au}$ as source, substrates and catalyst, respectively, SnTe nanoplates with lateral dimensions of $\sim 2-10 \mu \mathrm{m}$ were synthesized at relative low tempreatures $\left(\sim 300^{\circ} \mathrm{C}\right)$. Unfortunately, no topological surface properties were observed, likely because of the still thick thickness of 100-300 nm. Taking advantage of the vdWE method as we mentioned before, $\mathrm{Pb}_{1-x} \mathrm{Sn}_{x}$ Se nanoplates with thinner thickness of 20-25 nm were successfully synthesized by our group. ${ }^{[32,33]}$ However, their lateral dimension is only limited to 1-2 $\mu \mathrm{m}$, which makes it difficult to fabricate four terminal Hall devices. Very recently, using $\mathrm{PbTe} / \mathrm{SnTe}$ powders sources, we successfully grew $\mathrm{Pb}_{1-x} \mathrm{Sn}_{x} \mathrm{Te}$ nanoplates with maximum lateral size of $\sim 20 \mu \mathrm{m}$ and average thickness of $40 \mathrm{~nm}$ through CVD method. ${ }^{[9]}$ While growing, a (420) plane exposed $\mathrm{Pb}_{1-x} \mathrm{Sn}_{x} \mathrm{Te}$ nanobelt with width of $\sim 40 \mathrm{~nm}$ and length of $\sim 10 \mu \mathrm{m}$ initially formed on the $\mathrm{SiO}_{2} / \mathrm{Si}$ substrates. After that, the $\mathrm{Pb}_{1-x} \mathrm{Sn}_{x} \mathrm{Te}$ nuclei continued to grow along the vertical direction on the basis of the nanobelt because the lateral growth was limited by the $\mathrm{SiO}_{2} / \mathrm{Si}$ substrates which will induce big strain at the 
substrates/materials interfaces caused by the lattice/thermalmismatch. As a result, desired $2 \mathrm{D} \mathrm{Pb}_{1-x} \mathrm{Sn}_{x} \mathrm{Te}$ were obtained and able to be fabricated with Hall devices for the topological surface states characterization. As shown in Figure 11d, the stamp-assisted dry transfer method was used to fabricate the devices, based on which the angle- and temperature-dependent relationships between the change of magneto-conductance $(\Delta G)$ and magnetic field strength $(B)$ were studied. An obvious weak antilocalization (WAL) effect was observed at suitable temperature and angle of magnetic field (see Figure 11e).

For the time being, the study on 2D TCIs is just at its infancy. The above researches are just a primal demonstration of the possibility of synthesizing 2D TCIs through vdWE or CVD methods and there are such a plenty of unexplored issues that are remaining to be resolved. For example, even though the promising potential of vdWE method, almost none of the 2D TCIs has been achieved with thin enough thickness and large enough lateral sizes. In addition, as we mentioned before, the topological states of TCIs only rely on the high-symmetry crystalline surfaces and can be tuned by the compositions. Hence synthesizing 2D TCIs with tunable morphologies and compositions will definitely provide a platform to deeply study the underlying physical mechanism, which has never been studied.

\subsection{Other Applications}

Besides the four main applications discussed above, there are still other important applications based on 2D non-layered materials. As well known, surface plasmonic resonance (SPR) is a typical characteristic of noble metal nanostructures. One of current research attentions is to extend the SPR absorption to the near-infrared (NIR) range. ${ }^{[6]}$ Owing to the high aspect ratio, ultrathin $2 \mathrm{D}$ metal nanosheets show great opportunities to obtain ideal NIR SPR performance. For example, Zheng et al. achieved ultrathin Pd nanosheets with controllable edge length, tunable SPR absorption (826-1080 nm), and high stability. The Pd nanosheets demonstrate the potential ability for cancer therapy. ${ }^{[11]}$ In addition, for ferromagnetic metals, such as $\mathrm{Fe}, \mathrm{Co}$, and $\mathrm{Ni}$, their ultrathin 2D structures can enhance the spin and orbital moments. ${ }^{[81]}$ These ultrathin 2D ferromagnetic nanosheets may be utilized in miniaturized and high data density magnetic memory in the future.

For 2D non-layered semiconducting materials, constructing ultrathin heterostructures and corresponding electronic devices may be another potential application. For instance, similar to fabricated $\mathrm{GaAs}$ and $\mathrm{Al}_{x} \mathrm{Ga}_{1-x} \mathrm{As}$ by molecular beam epitaxial techniques, ${ }^{[82]}$ ultrathin non-layered 2D heterostrucutres can also be fabricated for the application in high-electron-mobility transistors.

Although various applications based on 2D non-layered materials have been realized and are being achieved, high performance new applications are still highly desired.

\section{Conclusion and Perspectives}

Holding novel physical properties, high flexibility and strong integration ability with Si-based electronic devices, 2D materials, including layered and non-layered materials, have demonstrated promising applications in optoelectronics, catalysis, energy conversion and storage, and topological crystalline insulators. In the past decade, various 2D layered materials, including metallic, semi-metallic, semiconducting and insulating materials, have been extensively studied. Motivated by the success of 2D layered materials, 2D non-layered materials are receiving growing research attentions. In this review, we summarize the recent progress on 2D non-layered materials with contents covering synthesis methods, properties and applications.

Controllable synthesis is always the first and critical step for any new materials. Dry synthesis strategies, such as CVD and vdWE growth methods, could be used to efficiently obtain 2D non-layered materials with high crystalline quality, free from contamination, and ultrathin thickness even below $10 \mathrm{~nm}$. However, it is still a great challenge to realize large-scale, thickness controllable, massive 2D material growth for the practical applications in the future. Especially, scaling down the thickness to few-atoms and obtaining uniform wafer-scale 2D nonlayered materials will be the next breakthrough in this area. In addition, since 2D non-layered materials can be in situ grown on $\mathrm{SiO}_{2} / \mathrm{Si}$ substrate by introducing a vdWE buffer layer, this may provide a possible method to realize the integration of $2 \mathrm{D}$ materials with Si-CMOS devices, which still remain unexplored and need further studies. On the other hand, wet chemical methods, such as SECS, TDS, CSS and CTS, are more thickness-controllable and can provide massive productions, making them suitable for catalysis as well as energy conversion and storage. However, new synthesis strategies are highly desired because 2D non-layered materials with larger sizes are preferred and there are still a lot of ultrathin materials that are not implemented.

At the time of writing, the whole research field on 2D non-layered materials is still at its beginning and most of the attentions are paid on materials synthesis. The situation that limited studies are on functional devices need to be changed in the near future. For example, since the properties of topological crystalline insulator are strongly dependent on the crystalline symmetry and the surface states, 2D non-layered materials may play a key role in this area. So far, the properties of $2 \mathrm{D}$ topological crystalline insulators have been investigated both by theories and experiments. On this basis, how to design and realize the functional devices will be a great challenge. Additionally, taking advantage of the 2D configuration, the combining of layered materials with non-layered materials and developing the hybrid devices may bring us new functions. Considering the novelty that 2D geometry has shown, it is worthwhile to see what surprises $2 \mathrm{D}$, especially ultrathin, non-layered materials would bring to us.

\section{Acknowledgements}

This work was supported by the National Natural Science Foundation of China (Nos. 21373065, 61474033 and 61574050), Strategic Priority Research Program of the Chinese Academy of Sciences (Grant No. XDA09040201), 973 Program of the Ministry of Science and Technology of China (No. 2012CB934103), and CAS Key Laboratory of Nanosystem 
and Hierarchical Fabrication. The authors also gratefully acknowledge the support of Youth Innovation Promotion Association CAS.

Received: June 29, 2016 Revised: July 25, 2016 Published online:

[1] S. Ithurria, M. D. Tessier, B. Mahler, R. P. S. M. Lobo, B. Dubertret, A. Efros, Nat. Mater. 2011, 10, 936.

[2] L. K. Li, G. J. Ye, V. Tran, R. X. Fei, G. R. Chen, H. C. Wang, J. Wang, K. Watanabe, T. Taniguchi, L. Yang, X. H. Chen, Y. B. Zhang, Nat. Nanotechnol. 2015, 10, 608.

[3] L. Li, F. Yang, G. J. Ye, Z. Zhang, Z. Zhu, W. Lou, X. Zhou, L. Li, K. Watanabe, T. Taniguchi, K. Chang, Y. Wang, X. H. Chen, Y. Zhang, Nat. Nanotechnol. 2016, 11, 593.

[4] X. X. Xi, L. Zhao, Z. F. Wang, H. Berger, L. Forro, J. Shan, K. F. Mak, Nat. Nanotechnol. 2015, 10, 765.

[5] Y. Ye, J. Xiao, H. Wang, Z. Ye, H. Zhu, M. Zhao, Y. Wang, J. Zhao, X. Yin, X. Zhang, Nat. Nanotechnol. 2016, 11, 598

[6] T. Ling, J. J. Wang, H. Zhang, S. T. Song, Y. Z. Zhou, J. Zhao, X. W. Du, Adv. Mater. 2015, 27, 5396.

[7] F. Wang, Z. X. Wang, Q. S. Wang, F. M. Wang, L. Yin, K. Xu, Y. Huang, J. He, Nanotechnology 2015, 26, 292001

[8] S. Z. Butler, S. M. Hollen, L. Y. Cao, Y. Cui, J. A. Gupta, H. R. Gutierrez, T. F. Heinz, S. S. Hong, J. X. Huang, A. F. Ismach, E. Johnston-Halperin, M. Kuno, V. V. Plashnitsa, R. D. Robinson, R. S. Ruoff, S. Salahuddin, J. Shan, L. Shi, M. G. Spencer, M. Terrones, W. Windl, J. E. Goldberger, ACS Nano 2013, 7, 2898.

[9] Q. S. Wang, K. M. Cai, J. Li, Y. Huang, Z. X. Wang, K. Xu, F. Wang, X. Y. Zhan, F. M. Wang, K. Y. Wang, J. He, Adv. Mater. 2016, 28, 617.

[10] C. L. Tan, H. Zhang, Nat. Commun. 2015, 6, 7873.

[11] X. Q. Huang, S. H. Tang, X. L. Mu, Y. Dai, G. X. Chen, Z. Y. Zhou, F. X. Ruan, Z. L. Yang, N. F. Zheng, Nat. Nanotechnol. 2011, 6, 28.

[12] X. Huang, S. Z. Li, Y. Z. Huang, S. X. Wu, X. Z. Zhou, S. Z. Li, C. L. Gan, F. Boey, C. A. Mirkin, H. Zhang, Nat. Commun. 2011, 2, 292.

[13] H. H. Duan, N. Yan, R. Yu, C. R. Chang, G. Zhou, H. S. Hu, H. P. Rong, Z. Q. Niu, J. J. Mao, H. Asakura, T. Tanaka, P. J. Dyson, J. Li, Y. D. Li, Nat. Commun. 2014, 5, 3093.

[14] T. Ling, J. J. Wang, H. Zhang, S. T. Song, Y. Z. Zhou, J. Zhao, X. W. Du, Adv. Mater. 2015, 27, 5396.

[15] Z. X. Fan, Y. H. Zhu, X. Huang, Y. Han, Q. X. Wang, Q. Liu, Y. Huang, C. L. Gan, H. Zhang, Angew. Chem. Int. Ed. 2015, 54, 5672.

[16] Z. X. Fan, X. Huang, Y. Han, M. Bosman, Q. X. Wang, Y. H. Zhu, Q. Liu, B. Li, Z. Y. Zeng, J. M. T. Wu, W. X. Shi, S. Z. Li, C. L. Gan, H. Zhang, Nat. Commun. 2015, 6, 6571.

[17] a) X. Y. Chen, Y. Zhou, Q. Liu, Z. D. Li, J. G. Liu, Z. G. Zou, ACS Appl. Mater. Interfaces 2012, 4, 3372; b) W. R. Cheng, J. F. He, T. Yao, Z. H. Sun, Y. Jiang, Q. H. Liu, S. Jiang, F. C. Hu, Z. Xie, B. He, W. S. Yan, S. Q. Wei, J. Am. Chem. Soc. 2014, 136, 10393.

[18] Y. T. Zhong, Y. J. Yang, Y. Ma, J. N. Yao, Chem. Commun. 2013, 49, 10355.

[19] F. C. Lei, Y. F. Sun, K. T. Liu, S. Gao, L. Liang, B. C. Pan, Y. Xie, J. Am. Chem. Soc. 2014, 136, 6826.

[20] Z. Q. Sun, T. Liao, Y. H. Dou, S. M. Hwang, M. S. Park, L. Jiang, J. H. Kim, S. X. Dou, Nat. Commun. 2014, 5, 3813

[21] Y. F. Sun, Q. H. Liu, S. Gao, H. Cheng, F. C. Lei, Z. H. Sun, Y. Jiang, H. B. Su, S. Q. Wei, Y. Xie, Nat. Commun. 2013, 4, 2899.

[22] C. Schliehe, B. H. Juarez, M. Pelletier, S. Jander, D. Greshnykh, M. Nagel, A. Meyer, S. Foerster, A. Kornowski, C. Klinke, H. Weller, Science 2010, 329, 550.
[23] Y. P. Du, Z. Y. Yin, J. X. Zhu, X. Huang, X. J. Wu, Z. Y. Zeng, Q. Y. Yan, H. Zhang, Nat. Commun. 2012, 3, 1177.

[24] a) S. Acharya, B. Das, U. Thupakula, K. Ariga, D. D. Sarma, J. Israelachvili, Y. Golan, Nano. Lett. 2013, 13, 409; b) Z. W. Wang, C. Schliehe, T. Wang, Y. Nagaoka, Y. C. Cao, W. A. Bassett, H. M. Wu, H. Y. Fan, H. Weller, J. Am. Chem. Soc. 2011, 133, 14484.

[25] G. B. Bhandari, K. Subedi, Y. F. He, Z. F. Jiang, M. Leopold, N. Reilly, H. P. Lu, A. T. Zayak, L. F. Sun, Chem. Mater. 2014, 26, 5433.

[26] Y. F. Sun, Z. H. Sun, S. Gao, H. Cheng, Q. H. Liu, J. Y. Piao, T. Yao, C. Z. Wu, S. L. Hu, S. Q. Wei, Y. Xie, Nat. Commun. 2012, 3, 1057.

[27] X. Y. Huang, J. Li, J. Am. Chem. Soc. 2007, 129, 3157.

[28] J. S. Son, X. D. Wen, J. Joo, J. Chae, S. I. Baek, K. Park, J. H. Kim, K. An, J. H. Yu, S. G. Kwon, S. H. Choi, Z. W. Wang, Y. W. Kim, Y. Kuk, R. Hoffmann, T. Hyeon, Angew. Chem. Int. Ed. 2009, 48, 6861.

[29] X. J. Wu, X. Huang, J. Q. Liu, H. Li, J. Yang, B. Li, W. Huang, H. Zhang, Angew. Chem. Int. Ed. 2014, 53, 5083.

[30] Q. S. Wang, F. Wang, J. Li, Z. X. Wang, X. Y. Zhan, J. He, Small 2015, 11, 4613.

[31] J. Shen, Y. Jung, A. S. Disa, F. J. Walker, C. H. Ahn, J. J. Cha, Nano Lett. 2014, 14, 4183.

[32] Q. S. Wang, K. Xu, Z. X. Wang, F. Wang, Y. Huang, M. Safdar, X. Y. Zhan, F. M. Wang, Z. Z. Cheng, J. He, Nano Lett. 2015, 15, 1183.

[33] Q. S. Wang, Y. Wen, F. R. Yao, Y. Huang, Z. X. Wang, M. L. Li, X. Y. Zhan, K. Xu, F. M. Wang, F. Wang, J. Li, K. H. Liu, C. Jiang, F. Q. Liu, J. He, Small 2015, 11, 5388.

[34] S. T. Ha, X. F. Liu, Q. Zhang, D. Giovanni, T. C. Sum, Q. H. Xiong, Adv. Opt. Mater. 2014, 2, 838.

[35] H. C. Cheng, G. M. Wang, D. H. Li, Q. Y. He, A. X. Yin, Y. Liu, H. Wu, M. N. Ding, Y. Huang, X. F. Duan, Nano Lett. 2016, 16, 367.

[36] L. Niu, X. F. Liu, C. X. Cong, C. Y. Wu, D. Wu, T. R. Chang, H. Wang, Q. S. Zeng, J. D. Zhou, X. L. Wang, W. Fu, P. Yu, Q. D. Fu, S. Najmaei, Z. H. Zhang, B. I. Yakobson, B. K. Tay, W. Zhou, H. T. Jeng, H. Lin, T. C. Sum, C. Jin, H. Y. He, T. Yu, Z. Liu, Adv. Mater. 2015, 27, 7800

[37] Q. S. Wang, M. Safdar, K. Xu, M. Mirza, Z. X. Wang, J. He, ACS Nano 2014, 8, 7497

[38] W. T. Bi, M. Zhou, Z. Y. Ma, H. Y. Zhang, J. B. Yu, Y. Xie, Chem. Commun. 2012, 48, 9162

[39] Y. Wen, Q. Wang, L. Yin, Q. Liu, F. Wang, F. Wang, Z. Wang, K. Liu, K. Xu, Y. Huang, T. A. Shifa, C. Jiang, J. Xiong, J. He, Adv. Mater. 2016, 28, 8051.

[40] Q. Wang, Y. Wang, P. He, L. Yin, Z. Wang, F. Wang, K. Xu, Y. Huang, F. Wang, C. Jiang, Jun He, Adv. Mater. 2016, 28, 6497.

[41] G. Wang, D. Li, H.-C. Cheng, Y. Li, C.-Y. Chen, A. Yin, Z. Zhao, Z. Lin, H. Wu, Q. He, M. Ding, Y. Liu, Y. Huang, X. Duan, Sci. Adv. 2015, 1, 9 .

[42] Q. Zhang, Y. X. Hu, S. R. Guo, J. Goebl, Y. D. Yin, Nano Lett. 2010, 10, 5037

[43] X. Yin, X. H. Liu, Y. T. Pan, K. A. Walsh, H. Yang, Nano Lett. 2014, $14,7188$.

[44] C. P. Hou, J. Zhu, C. Liu, X. Wang, Q. Kuang, L. S. Zheng, Crystengcomm 2013, 15, 6127.

[45] A. X. Yin, W. C. Liu, J. Ke, W. Zhu, J. Gu, Y. W. Zhang, C. H. Yan, J. Am. Chem. Soc. 2012, 134, 20479.

[46] a) X. Q. Huang, S. H. Tang, B. J. Liu, B. Ren, N. F. Zheng, Adv. Mater. 2011, 23, 3420; b) M. Chen, S. H. Tang, Z. D. Guo, X. Y. Wang, S. G. Mo, X. Q. Huang, G. Liu, N. F. Zheng, Adv. Mater. 2014, 26, 8210.

[47] F. Wang, J. H. Seo, G. F. Luo, M. B. Starr, Z. D. Li, D. L. Geng, X. Yin, S. Y. Wang, D. G. Fraser, D. Morgan, Z. Q. Ma, X. D. Wang, Nat. Commun. 2016, 7, 10444. 
[48] S. J. Ding, D. Y. Luan, F. Y. C. Boey, J. S. Chen, X. W. Lou, Chem. Commun. 2011, 47, 7155.

[49] Z. F. Huang, A. N. Zhou, J. F. Wu, Y. Q. Chen, X. L. Lan, H. Bai, L. Li, Adv. Mater. 2016, 28, 1703.

[50] Y. Q. Zhu, C. B. Cao, S. Tao, W. S. Chu, Z. Y. Wu, Y. D. Li, Sci. Rep. 2014, 4, 5787 .

[51] J. B. Zhu, L. F. Bai, Y. F. Sun, X. D. Zhang, Q. Y. Li, B. X. Cao, W. S. Yan, Y. Xie, Nanoscale 2013, 5, 5241.

[52] Q. Zhang, B. Yan, Chem.-Eur. J. 2012, 18, 5150.

[53] T. Bielewicz, S. Dogan, C. Klinke, Small 2015, 11, 826.

[54] S. Lee, D. T. Lee, J. H. Ko, W. J. Kim, J. Joo, S. Jeong, J. A. McGuire, Y. H. Kim, D. C. Lee, RSC. Adv. 2014, 4, 9842.

[55] C. Wang, Y. Zhou, M. Y. Ge, X. B. Xu, Z. L. Zhang, J. Z. Jiang, J. Am. Chem. Soc. 2010, 132, 46.

[56] H. W. Huang, Q. Yu, X. S. Peng, Z. Z. Ye, Chem. Commun. 2011, 47, 12831.

[57] X. D. Zhang, J. J. Zhang, J. Y. Zhao, B. C. Pan, M. G. Kong, J. Chen, Y. Xie, J. Am. Chem. Soc. 2012, 134, 11908.

[58] A. Kudo, Y. Miseki, Chem. Soc. Rev. 2009, 38, 253.

[59] Y. Jiao, Y. Zheng, M. T. Jaroniec, S. Z. Qiao, Chem. Soc. Rev. 2015, 44, 2060.

[60] A. Fujishima, K. Honda, Nature 1972, 238, 37.

[61] Y. Xu, W. W. Zhao, R. Xu, Y. M. Shi, B. Zhang, Chem. Commun. 2013, 49, 9803.

[62] F. Wang, Y. Li, T. A. Shifa, K. Liu, F. Wang, Z. Wang, P. Xu, Q. Wang, J. He, Angew. Chem. Int. Ed. 2016, 55, 6919.

[63] Y. F. Sun, F. C. Lei, S. Gao, B. C. Pan, J. F. Zhou, Y. Xie, Angew. Chem. Int. Ed. 2013, 52, 10569.

[64] a) F. M. Wang, X. Y. Zhan, Z. Z. Cheng, Z. X. Wang, Q. S. Wang, K. Xu, M. Safdar, J. He, Small 2015, 11, 749; b) X. H. Lu, T. Zhai, X. H. Zhang, Y. Q. Shen, L. Y. Yuan, B. Hu, L. Gong, J. Chen, Y. H. Gao, J. Zhou, Y. X. Tong, Z. L. Wang, Adv. Mater. 2012, 24, 938; c) F. M. Wang, Y. C. Li, Z. Z. Cheng, K. Xu, X. Y. Zhan, Z. X. Wang, J. He, Phys. Chem. Chem. Phys. 2014, 16, 12214.

[65] S. Gao, Y. F. Sun, F. C. Lei, L. Liang, J. W. Liu, W. T. Bi, B. C. Pan, Y. Xie, Angew. Chem. Int. Ed. 2014, 53, 12789.

[66] J. S. Chen, T. Zhu, X. H. Yang, H. G. Yang, X. W. Lou, J. Am. Chem. Soc. 2010, 132, 13162

[67] a) J. S. Chen, Y. L. Tan, C. M. Li, Y. L. Cheah, D. Y. Luan, S. Madhavi, F. Y. C. Boey, L. A. Archer, X. W. Lou, J. Am. Chem. Soc. 2010, 132, 6124; b) H. S. Liu, Z. H. Bi, X. G. Sun, R. R. Unocic, M. P. Paranthaman, S. Dai, G. M. Brown, Adv. Mater. 2011, 23, 3450.

[68] a) J. H. Liu, J. S. Chen, X. F. Wei, X. W. Lou, X. W. Liu, Adv. Mater. 2011, 23, 998; b) Y. Ren, A. R. Armstrong, F. Jiao, P. G. Bruce, J. Am. Chem. Soc. 2010, 132, 996.

[69] a) Z. Y. Yin, H. Li, H. Li, L. Jiang, Y. M. Shi, Y. H. Sun, G. Lu, Q. Zhang, X. D. Chen, H. Zhang, ACS Nano 2012, 6, 74; b) O. Lopez-Sanchez, D. Lembke, M. Kayci, A. Radenovic, A. Kis, Nat. Nanotechnol. 2013, 8, 497.

[70] M. C. Beard, J. M. Luther, O. E. Semonin, A. J. Nozik, Acc. Chem. Res. 2013, 46, 1252.

[71] M. Aerts, T. Bielewicz, C. Klinke, F. C. Grozema, A. J. Houtepen, J. M. Schins, L. D. A. Siebbeles, Nat. Commun. 2014, 5, 3789.

[72] a) S. D. Stranks, G. E. Eperon, G. Grancini, C. Menelaou, M. J. P. Alcocer, T. Leijtens, L. M. Herz, A. Petrozza, H. J. Snaith, Science 2013, 342, 341; b) M. A. Green, A. Ho-Baillie, H. J. Snaith, Nat Photonics 2014, 8, 506; c) G. C. Xing, N. Mathews, S. Y. Sun S. S. Lim, Y. M. Lam, M. Gratzel, S. Mhaisalkar, T. C. Sum, Science 2013, 342, 344.

[73] W. S. Yang, J. H. Noh, N. J. Jeon, Y. C. Kim, S. Ryu, J. Seo, S. I. Seok, Science 2015, 348, 1234.

[74] P. Gao, M. Gratzel, M. K. Nazeeruddin, Energy Environ. Sci. 2014, 7, 2448.

[75] D. Cheyns, J. Poortmans, P. Heremans, C. Deibel, S. Verlaak, B. P. Rand, J. Genoe, Phys. Rev. B 2008, 77.

[76] a) Y. Ando, J Phys Soc Jpn 2013, 82; b) X. L. Qi, S. C. Zhang, Phys. Today 2010, 63, 33.

[77] L. A. Fu, Phys. Rev. Lett. 2011, 106

[78] T. H. Hsieh, H. Lin, J. W. Liu, W. H. Duan, A. Bansil, L. Fu, Nat Commun. 2012, 3, 982.

[79] P. Dziawa, B. J. Kowalski, K. Dybko, R. Buczko, A. Szczerbakow M. Szot, E. Lusakowska, T. Balasubramanian, B. M. Wojek, M. H. Berntsen, O. Tjernberg, T. Story, Nat. Mater. 2012, 11, 1023.

[80] a) Y. Tanaka, Z. Ren, T. Sato, K. Nakayama, S. Souma, T. Takahashi, K. Segawa, Y. Ando, Nat. Phys. 2012, 8, 800; b) S. Y. Xu, C. Liu, N. Alidoust, M. Neupane, D. Qian, I. Belopolski, J. D. Denlinger, Y. J. Wang, H. Lin, L. A. Wray, G. Landolt, B. Slomski, J. H. Dil, A. Marcinkova, E. Morosan, Q. Gibson, R. Sankar, F. C. Chou, R. J. Cava, A. Bansil, M. Z. Hasan, Nat. Commun. 2012, 3, 1192.

[81] O. Hjortstam, J. Trygg, J. M. Wills, B. Johansson, O. Eriksson, Phys. Rev. B 1996, 53, 9204.

[82] a) Q. H. Wang, K. Kalantar-Zadeh, A. Kis, J. N. Coleman M. S. Strano, Nat. Nanotechnol. 2012, 7, 699; b) M. A. Khan, A. Bhattarai, J. N. Kuznia, D. T. Olson, Appl. Phys. Lett. 1993, 63, 1214.

[83] S. Gao, Y. Lin, X. C. Jiao, Y. F. Sun, Q. Q. Luo, W. H. Zhang, D. Q. Li, J. L. Yang, Y. Xie, Nature 2016, 529, 68.

[84] Y. W. Liu, H. Cheng, M. J. Lyu, S. J. Fan, Q. H. Liu, W. S. Zhang, Y. D. Zhi, C. M. Wang, C. Xiao, S. Q. Wei, B. J. Ye, Y. Xie, J. Am. Chem. Soc. 2014, 136, 15670.

[85] P. Z. Chen, K. Xu, X. L. Li, Y. Q. Guo, D. Zhou, J. Y. Zhao, X. J. Wu, C. Z. Wu, Y. Xie, Chem. Sci. 2014, 5, 2251.

[86] Z. Y. Lu, J. X. Zhu, D. H. Sim, W. W. Zhou, W. H. Ship, H. H. Hng, Q. Y. Yan, Chem. Mater. 2011, 23, 5293. 